Computational and Mathematical Methods in Medicine

Vol. 10, No. 1, March 2009, 9-38

Taylor \& Francis

Taylor \& Francis Group

\title{
Mathematical modelling of immune response in tissues
}

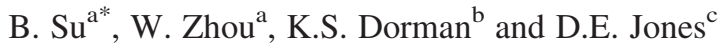 \\ ${ }^{a}$ Department of Mathematics, Iowa State University, Ames, IA 50010, USA; ${ }^{b}$ Department of Genetics \\ Development and Cell Biology, Iowa State University, Ames, IA 50010, USA; ${ }^{c}$ Department of \\ Veterinary Pathology, Iowa State University, Ames, IA 50010, USA
}

(Received 14 December 2007; final version received 11 January 2008)

\begin{abstract}
We have developed a spatial-temporal mathematical model (PDE) to capture fundamental aspects of the immune response to antigen. We have considered terms that broadly describe intercellular communication, cell movement, and effector function (activation or inhibition). The PDE model is robust to variation in antigen load and it can account for (1) antigen recognition, (2) an innate immune response, (3) an adaptive immune response, (4) the elimination of antigen and subsequent resolution of the immune response or (5) equilibrium of the immune response to the presence of persistent antigen (chronic infection) and the formation of a granuloma.
\end{abstract}

Keywords: immune system; biological sensor; regulatory mechanism; chemotaxis process; homogenization; numerical simulation

AMS Subject Classification: 35M10; 35K55; 92B05; 92C37; 92C50

\section{Introduction}

The majority of infectious diseases trigger an immune response by introducing foreign antigen and a secondary signal, usually molecules that indicate a foreign presence or tissue damage, that together warn of an impending threat to the host. Once initiated, a successful immune response must balance the pro-inflammatory, destructive actions of immune effector cells with the protection of organ function. This balance becomes particularly critical when battling pathogens that manage to persist despite recognition by and stimulation of the immune system. If the pathogen does not immediately disseminate, a classic immune strategy is to wall it off inside a structured granuloma [27], whose formation is itself a highly regulated process. The mechanics of balancing pro- vs. anti-inflammatory actions and building granuloma-like structures are dependent upon multiple factors regulating cell trafficking, cell-cell communication and effector function [27]. With multitudes of cell signals and cell signalling events, it is difficult to develop a coherent model of the hostpathogen relationship that is still simple enough to link with available knowledge and data.

Our goal was to build a partial differential equation (PDE) model identifying a minimal set of immune factors that would recapitulate the host immune response to persistent localized infection and the patterned immune response of granuloma formation. Naturally, pathogens have developed many mechanisms to interfere or manipulate these patterned host responses. To avoid, at least at first, the complexity of such pathogen/host interactions, we consider the simplest immune trigger: foreign antigen combined with

\footnotetext{
ISSN 1748-670X print/ISSN 1748-6718 online (C) 2009 Taylor \& Francis DOI: $10.1080 / 17486700801982713$

http://www.informaworld.com
}

*Corresponding author. Email: bosu@iastate.edu; bosu@mis.mpg.de 
pathogen associated molecular patterns (PAMPs, Ref. Section 2.1) [41,43]. The immune system we model includes all the basic factors: antigen, immune cells, chemokines and cytokines. Dendritic cells turn out to be an essential component of the model, serving as a biological sensor that detects PAMPs. Activated by the dendritic cells, T cells ultimately move toward the site of infection in the well-established temporal order of granuloma formation [27,59]. We also find a requirement for some kind of regulatory mechanism, still rather rudimentary in our model, to limit the immune response and avoid unlimited inflammation during persistent infection.

The paper is organized as follows. Section 2 is a detailed description of our partial differential equation model for the immune response in tissue. In Section 3, we select and justify model parameters. Section 4 is a brief discussion of the numerical simulation approach used to solve the model proposed in Section 2. In Section 5, we show the results of numerical experiments to test our model and define the minimal set of immune factors. Finally, discussion and conclusions about the model are made in Section 6.

\section{The mathematical model}

The immune response follows a pattern of engagement with foreign antigen: recognition, initiation, effector response and resolution or equilibration to a new steady state [27]. The PDE model we develop captures all of these stages of the immune response. In the model, antigen and secondary signal diffuse from either a transient or persistent source. The abundance of responsive neutrophils allows their rapid deployment and early domination of the infection site, where they quickly reduce the amount of antigen and establish an initial gradient of chemokine [49]. A troublesome side effect of their activity is a potpourri of dangerous chemicals designed to destroy the pathogen, but often damaging the host as well. To launch a more regulated immune attack, both macrophages and dendritic cells then migrate toward the antigen source via the chemokine gradient [26]. Dendritic cells uptake antigen and activate upon PAMPs recognition, undergo maturation and migrate away from the chemokine source to the model boundary. The boundary of our symmetric simulation area $\Omega$ serves as a surrogate for the lymph nodes, whose most important role is to develop an adaptive immune response. Mature dendritic cells arriving in the lymph nodes activate effector T cells, which in turn become sensitive to the chemokine gradient and migrate to the antigen/PAMP source $[37,44]$. Once at the site of infection, effector $\mathrm{T}$ cells oversee immune maturation by producing cytokines that activate local macrophages [27]. Activated macrophages process additional antigens/PAMPs and limit neutrophil-induced damage by blocking further neutrophil recruitment [1,23]. Activated macrophages are less reckless than neutrophils, but they contribute to the pro-inflammatory environment that can damage the host in the long run. To limit the damage, regulatory $\mathrm{T}$ cells are also recruited to the site of infection, where they produce an inhibitory cytokine that can block activation of macrophages and limit macrophage-induced tissue damage $[5,8]$. With regulatory $\mathrm{T}$ cells recruited at the right time, a comprehensive self-regulation system is established to cope with both acute and chronic pathogen infections. A complete list of state variables involved in the model is found in Table 1.

Due to the large number of parameters, establishing consistent notation can be challenging. In the equations that follow, we have used the same greek letter to represent parameters with similar meaning. We use $\beta$ for secretion, $\gamma$ for downregulation, $\delta$ for activation, $\lambda$ for uptake, $\mu$ for death/decay, and $\epsilon$ for threshold parameters. The parameter subscripts are chosen to indicate which variable is affected by the parameter, so $\mu_{N}$ affects neutrophil numbers. When more than one variable can affect another variable via the same 
Table 1. State variables of model system.

\begin{tabular}{ll}
\hline & Immune cells \\
\hline$A$ & Antigens/PAMP \\
$D E$ & Immature dendritic cells \\
$D A$ & Mature dendritic cells \\
$T$ & Effector T cells \\
$T_{r e g}$ & Regulatory T cells \\
$M R$ & Resting macrophages \\
$M A$ & Activated macrophages \\
$N$ & Neutrophils \\
$N D$ & Apoptotic neutrophils \\
\hline & Cytokine \\
\hline$C M$ & Cytokine secreted by macrophages after engulfing apoptotic neutrophils \\
$C T$ & Effector T cell-released cytokines \\
$C T_{r e g}$ & Regulatory T cell-released cytokines \\
$C H$ & Chemokines \\
\hline
\end{tabular}

mechanism, or when greater clarification is desired, the subscript $X \mid Y$ indicates variable $X$ is affected via the action of $Y$. For example, $\lambda_{A \mid N}$ and $\lambda_{A \mid M A}$ are the uptake rates of antigen by neutrophil and macrophage.

\subsection{Antigen and PAMP}

The immune system has developed specific pattern recognition receptors (PRRs) to detect a broad range of ligands, termed pathogen associated molecular patterns (PAMPs), found on infectious agents [73]. PRRs are found on cells throughout the immune system but are best described on cells of the innate immune system, including neutrophils, macrophages and dendritic cells [4]. PAMPs include bacterial flagellin, lipopolysaccharide (LPS), bacterial DNA sequences and double stranded RNA. The immune system we model is a simplified version triggered only by antigen (e.g. ovalbumin) and PAMP molecules (e.g. LPS). We assume that the antigen and PAMP molecules share similar dynamics, produced at the same rate $g(x, t)$, degrading at the same constant rate $\mu_{A}$, diffusing at roughly the same rate $D_{A}$, as justified by the Stokes-Einstein equation [25], and consumed largely through the indiscriminate process of pinocytosis by macrophages, neutrophils and immature dendritic cells. Henceforth, we treat both molecules as one substance, denoted by $A$, that satisfies the following partial differential equation, along with initial and boundary conditions.

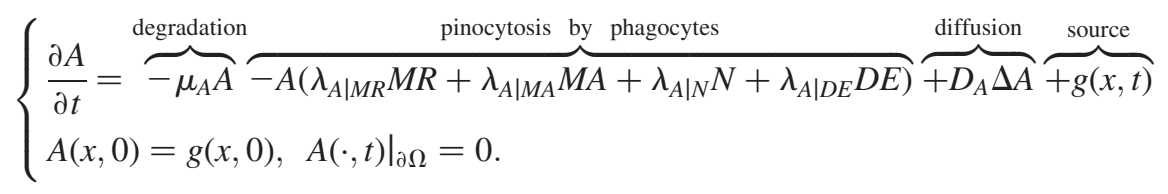

In the equation, $\lambda_{M R}, \lambda_{M A}, \lambda_{N}$ and $\lambda_{D E}$ are the rates of antigen/PAMP uptake through pinocytosis by resting macrophages $(M R)$, activated macrophages $(M A)$, neutrophils $(N)$ and immature dendritic cells $(D E)$. The antigen/PAMP source $g(x, t)$ maybe transient, representing acute infection or constant, to represent a persistent infection, such as a resistant, replicating pathogen. We assume the model region is sufficiently large so that antigen levels at the boundary remain negligible. 


\subsection{Neutrophils and apoptotic neutrophils}

Neutrophils, denoted by $N$, constitute $40-70 \%$ of the white blood cells in the blood stream [7]. They respond early to threats against the host by detecting changes in the vascular endothelium induced by tissue damage and/or infection [27]. Neutrophils, and other immune cells, initially approach the source of antigen through the microvascular system that extends into the tissue. Once activated by changes in the vascular endothelium, neutrophils exit the microvasculature and move through the tissue by sensing molecules, or chemokines, produced by damaged or infected tissue [1,27]. This process, termed chemotaxis, plays a significant role in immune cell motion in our model [57].

Neutrophil's movement is governed by Stokes equation in blood vessels and the considerably slower processes of diffusion and chemotaxis in the tissue [10,28]. We account for movement through the microvasculature using the concept of homogenization. Homogenization is a sophisticated, powerful tool to study macroscopic (large scale) behaviour of microscopic (small scale) heterogeneous materials [12,68,70]. For simplicity, we assume that the microvasculature tubules form a reticulated structure, densely distributed everywhere and with arbitrary direction. With these assumptions, homogenization is applied to the mix of microvasculature and extravasculature tissue to obtain an effective diffusion/chemotaxis process, where the diffusion coefficient $D_{N}$ or chemotaxis coefficient of neutrophils, for example, is substantially larger than diffusion/chemotaxis coefficients measured in vitro [46] while chemotaxis coefficient is in the same range as that measured in in vitro. Hereafter, the motion of most immune cells in the tissue is justified as effective diffusion or effective chemotaxis.

One of the main tasks of neutrophils is to deliver a potent mix of noxious chemicals to the presumed location of the invading pathogens. The delivery is achieved through a process called degranulation, which culminates in the death of the neutrophil $[1,49]$. It should not be surprising that such a lethal arsenal is highly regulated, mostly to ensure that the chemicals are released near pathogens. It would be wasteful, not to mention dangerous, to release the chemicals in the absence of pathogen. In our model, we use the successful scavenging of antigen/PAMP as an indication of the presence of pathogen, so antigen uptake at rate $\lambda_{A \mid N}$ is immediately followed by neutrophil death by degranulation. If neutrophils do not encounter antigen/PAMP after leaving the microsvasculature, they die through a constitutive process of apoptosis at rate $\mu_{N}$, a programmed cell death that is much cleaner and less destructive to surrounding tissue (Figure 1).

Degranulation of neutrophils, no matter how targeted, is nevertheless destructive to surrounding host tissue, so there is heavy incentive to reduce the number of neutrophils

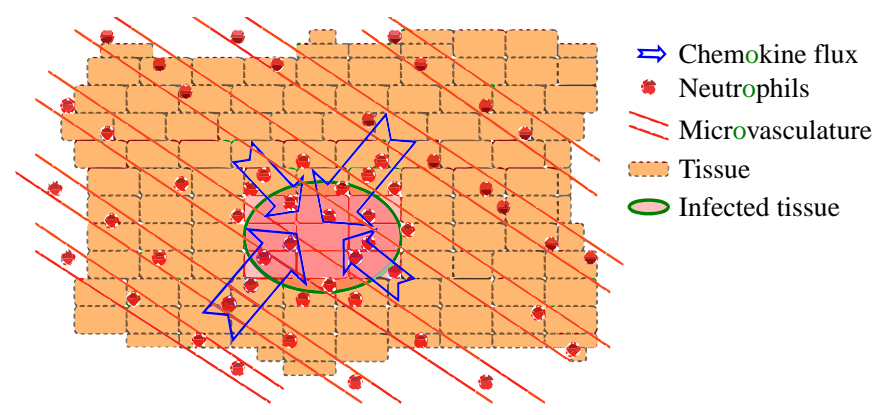

Figure 1. Schematic diagram of neutrophil movement in microvascularized tissue. 
as soon as possible. The presence of apoptotic neutrophils, represented by ND in our model, serves as an early indication of the successful clearance of pathogen $[1,49]$. Apoptotic cells are cleared through phagocytosis at rate $\lambda_{N D \mid M A}$ by activated macrophages $M A$. Upon phagocytosing apoptotic neutrophils, activated macrophages hasten to limit the damage of future degranulation by producing a chemokine $C M$ [see Equation (9)] that stops the influx of fresh neutrophils into the model area [1,9], implemented as a on/off boundary condition thresholded at level $\epsilon_{N}$.

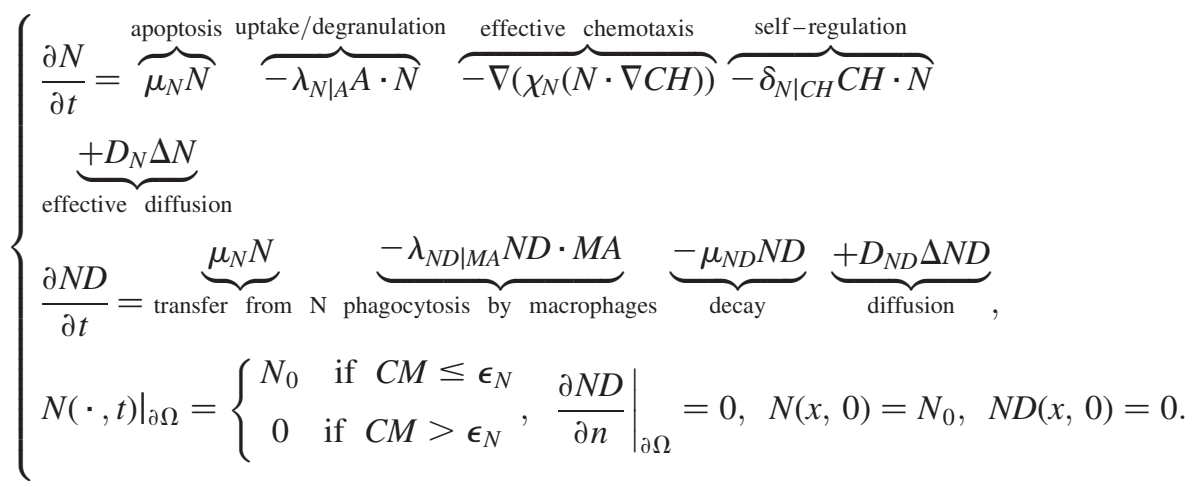

The convection-diffusion and reaction-diffusion equations capturing these fundamental characteristics of neutrophil and apoptotic neutrophil behaviour are shown below, along with boundary and initial conditions. Because we assume a high density of microvasculature everywhere in the tissue, we assume the initial concentration of neutrophils is constant throughout the simulation domain, though initially there are no apoptotic neutrophils. Finally, we introduce a self-regulation term $\delta_{N \mid C H} \mathrm{CH} \cdot \mathrm{N}$, involving the generic chemokine $\mathrm{CH}$ (see Section 2.6). Neutrophils are known to release many self-regulating molecules $[14,54,77]$. Since chemokine $\mathrm{CH}$ is predominately produced by neutrophils early in infection [see Equation (9)], we use $\mathrm{CH}$ levels to auto-regulate these cells.

\subsection{Dendritic cells}

Some time after the innate immune response launches at the infection site, evidence of the infection reaches the lymph nodes via antigen presenting cells (APCs). The adaptive immune response is a carefully orchestrated affair that detects the pathogen with greater sensitivity and, deflecting unwanted damage to the host, directs the inflammatory response more precisely at the pathogen. We consider dendritic cells to be the main antigen presenting cells (APCs), with two phases: immature and mature [36].

Immature dendritic cells, $D E$ in Equation (4), migrate toward the site of infection following the chemokine gradient established by neutrophils and, later, activated macrophages. The immature dendritic cells engulf antigens/PAMPs, contributing to one of the processing terms in Equation (1), and degrade them into peptides [37]. Simultaneously, immature dendritic cells undergo PAMP-induced activation to become mature dendritic cells, DA. Mature dendritic cells reverse responsiveness to the chemokine gradient and migrate away from the antigen/PAMP source toward the lymph 
nodes. As an approximation, we assume the activation rate

$$
\gamma_{D E}(A)=\left\{\begin{array}{cc}
\gamma_{D E} & A>\varepsilon_{D E} \\
0 & A \leq \varepsilon_{D E}
\end{array}\right.
$$

is a threshold function, which as simply as possible, captures the fact that activation of dendritic cells depends on the amount of antigen and PAMP [79]. Dendritic cells also experience natural death at degradation rate $\mu_{D E}$ or $\mu_{D A}$ and effective diffusion with coefficient $D_{D E}$ or $D_{D A}$ (Figure 2)

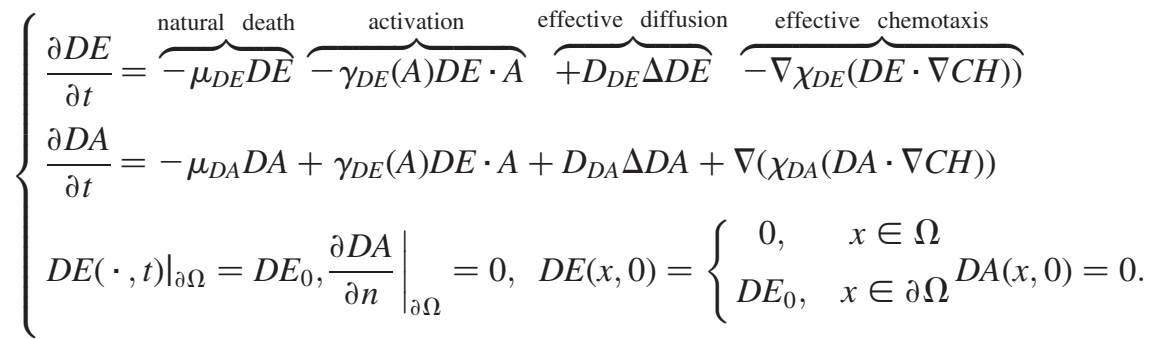

\subsection{Effector $T$ cells and regulatory $T$ cells}

There are a myriad of factors involved in immune regulation, and how they actually achieve the pro- and anti-inflammatory balance is poorly understood. For simplicity, we do not distinguish among the many varieties of effector T cells. In our simple model, the common function of all these effector $\mathrm{T}$ cell subsets is to reduce antigen load

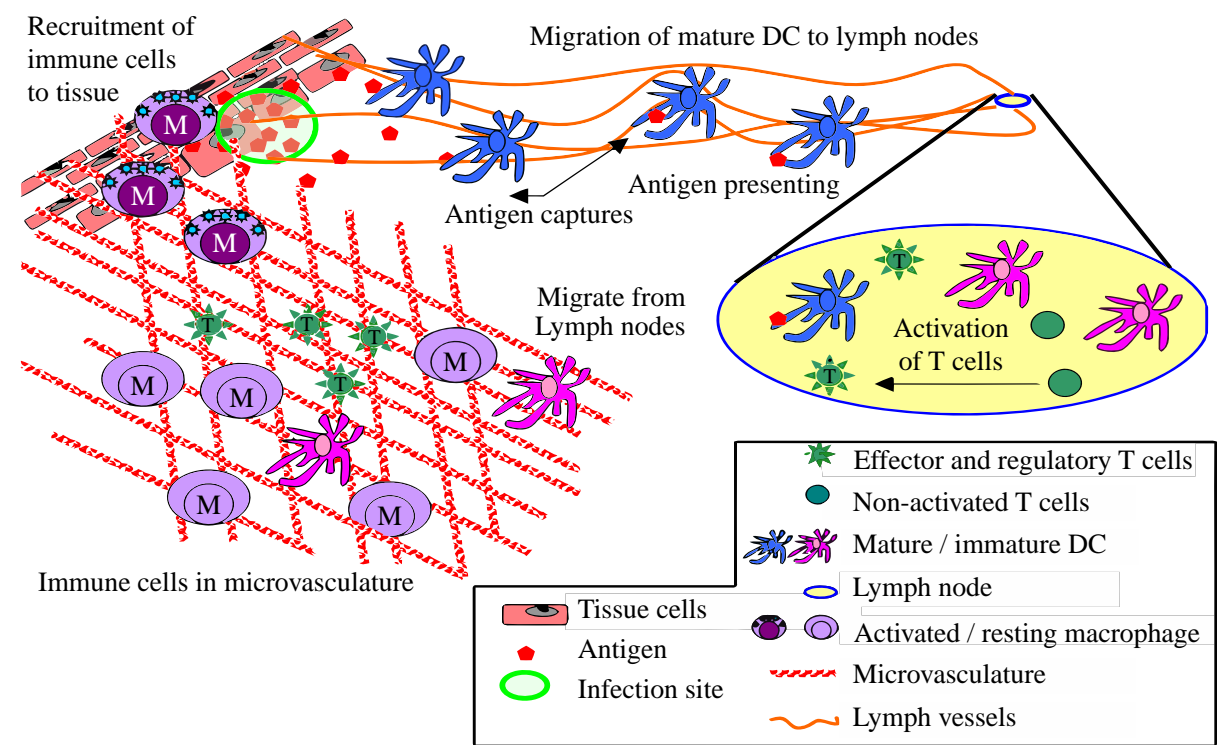

Figure 2. Comprehensive schematic diagram of immune system in our PDE model. DC denotes dendritic cells. 
by stimulating the immune response through pro-inflammatory signals. In contrast, the regulatory $\mathrm{T}$ cells and their variants [22] function to reduce inflammation. In addition, we only track the effector and regulatory $\mathrm{T}$ cells that are responsive to the delivered antigen, so all $\mathrm{T}$ cells we consider can be activated by APC circulating from the antigen/PAMP source.

\subsubsection{Effector $T$ cells}

Naïve $\mathrm{T}$ cells continuously recirculate between the vasculature and lymph nodes. In the lymph nodes, they interact with dendritic cells, looking for presented antigen that binds their T cell receptor [27]. If such antigen is found, they migrate in an activated state to the site of infection via the microvasculature. We simplify the model by assuming that naive $\mathrm{T}$ cells reside in the lymph node (the boundary) until they are activated by contact with the mature dendritic cells DA of Equation (4). The activated effector T cells have no cytotoxic activities, but divide rapidly, migrate to the source of antigen/PAMP, and secrete proinflammatory cytokines to upregulate the immune response, particularly via the macrophage population $[19,27]$. The modelling of these cytokines and their impact on macrophages is discussed in Sections 2.5 and 2.6 (Figure 3).

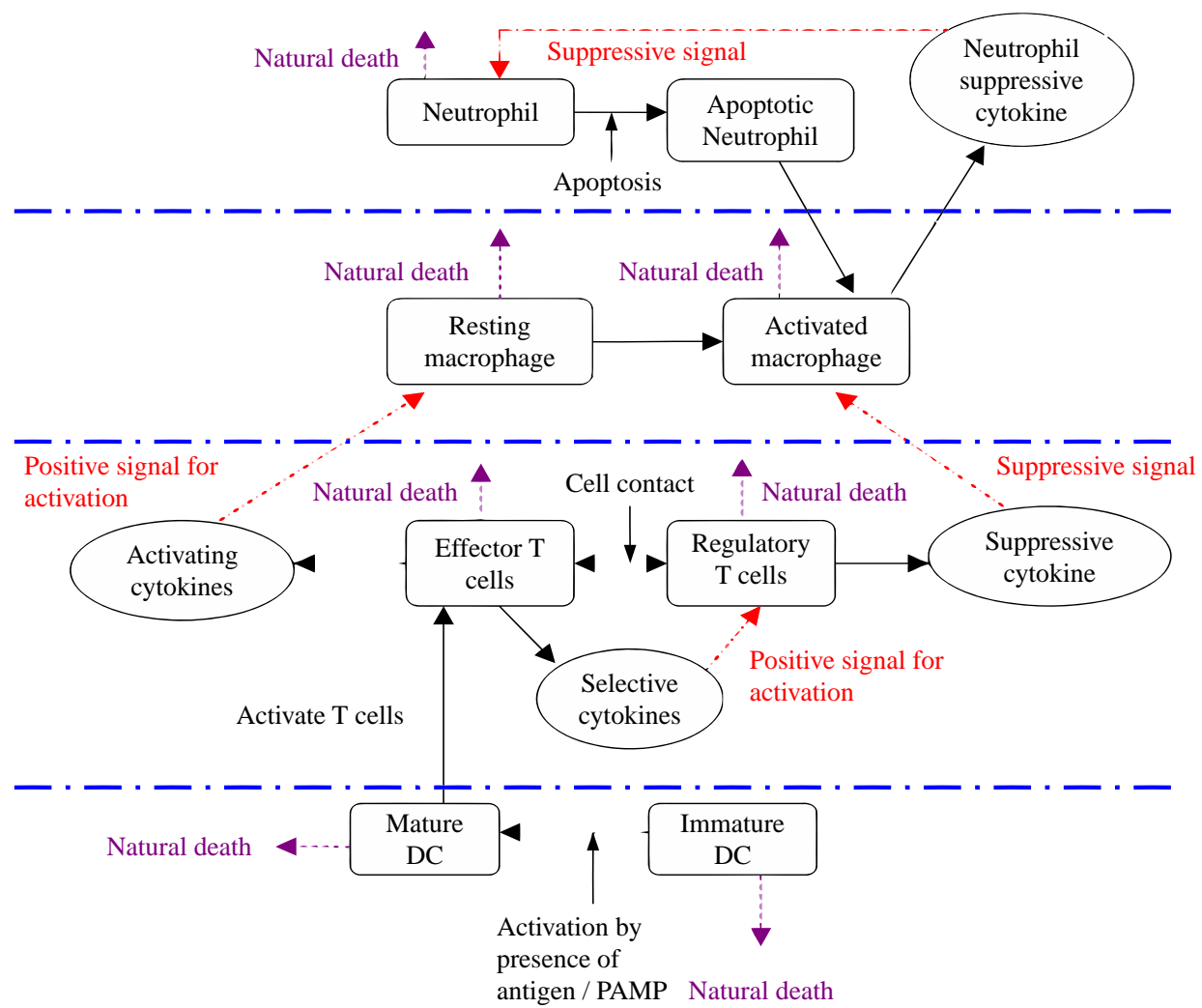

Figure 3. Regulatory mechanisms involved in the model. As discussed in Section 2.4, effector and regulatory $\mathrm{T}$ cells play a central regulatory role in the immune system, interacting directly with each other and secreting cytokines that manage macrophage activation. Furthermore, as per Sections 2.3 and 2.4, mature dendritic cells help regulate $\mathrm{T}$ cell recruitment. 


\subsubsection{Regulatory $T$ cells}

Recently, a subpopulation of $\mathrm{T}$ cells that play a significant role in limiting the proinflammatory immune response has been discovered $[27,69]$. The mechanisms by which these cells, called regulatory $\mathrm{T}$ cells, exert their suppressory/regulatory activities is not completely understood, but it may be mediated by both immunosuppressive cytokines, such as TGF- $\beta$ and IL-10, as well as direct cell-to-cell contact with T effector cells $[29,62,75]$. The term $-\delta_{T} T \cdot T_{\text {reg }}$ in Equation (5) accounts for possible contact-mediated suppression, where regulatory $\mathrm{T}$ cells directly abrogate effector $\mathrm{T}$ cell function, effectively removing such cells from the infection site. Also, cytokines produced by regulatory T cells (Section 2.6) affect macrophages, which synthesize cytokine signals from both effector and regulatory $\mathrm{T}$ cells in order to determine their activation status, as discussed in Section 2.5.

As a summary, in our model:

1. Regulatory $\mathrm{T}$ cells suppress primed effector $\mathrm{T}$ cells through direct cell contact at a constant rate, and this contributes to effector $\mathrm{T}$ cells becoming unresponsive;

2. Regulatory $\mathrm{T}$ cells suppress the immune response by secreting undefined negative signals, which we discuss in Section 2.6 and denote by $C T_{\text {reg }}$;

3. Regulatory $\mathrm{T}$ cells undergo chemotaxis and diffusion in a manner similar to previously discussed effector $\mathrm{T}$ cells.

\subsubsection{Recruitment and initial distribution of effector and regulatory $T$ cells}

To complete the modelling of $\mathrm{T}$ cells, we need to specify boundary and initial conditions [summarized in Equation (5)]. Here, the role of the boundary as lymph node is pertinent. As discussed in Section 2.3, there are no effector T cells until mature dendritic cells presenting antigen arrive in the lymph nodes (boundary), but as long as mature dendritic cells (APC) are at the lymph node, effector T cells appear on the boundary at rate $\beta_{D A}$ per APC.

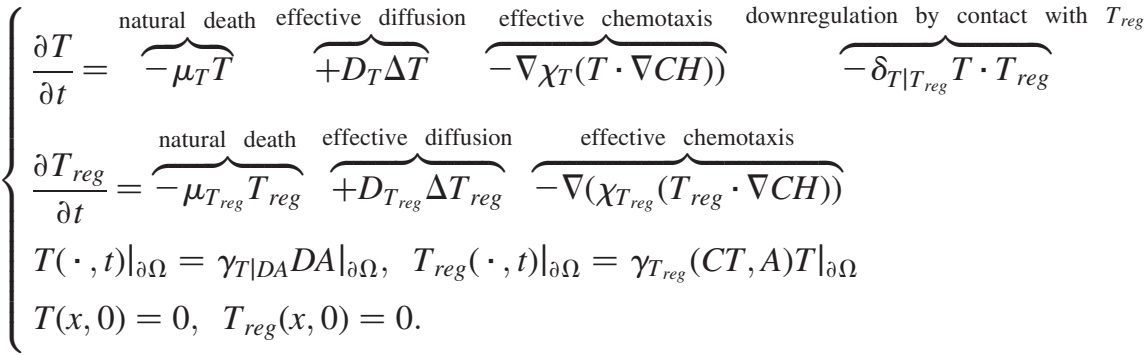

$$
\begin{aligned}
& \gamma_{T_{\text {reg }}}(C T, A)= \begin{cases}\gamma_{T_{\text {reg }}} T, & \left.C T(\cdot, t)\right|_{\partial \Omega}>\epsilon_{T_{\text {reg }}}^{1}+\epsilon_{T_{\text {reg }}}^{2} \int_{\Omega} A(x, t) \mathrm{d} x \\
0, & \text { otherwise }\end{cases}
\end{aligned}
$$

Less is known about the origins of regulatory $\mathrm{T}$ cells, and there may be several different sources, depending upon the specific immune response [29,33,34,69,75]. In our model, they begin life as effector $\mathrm{T}$ cells in the lymph node that become activated by signals from the infection site [69]. We assume this activation signal originates with the activated effector $T$ cell population itself, which proliferates and secretes positive growth signals, like IL-2 [30,34], that promote regulatory T cell differentiation and survival [22]. However, immune suppression is only warranted when immune inflammation exceeds 
the perceived threat level. The severity of the threat is proportionally related to the total amount of antigen/PAMP, or antigen load. To summarize these considerations, we assume development of regulatory $\mathrm{T}$ cells in the lymph node depends on a threshold function $h(C T, A)$ defined in Equation (6). This threshold function uses a line, with constants $\epsilon_{T_{r e e}}^{1}$ and $\epsilon_{T_{\mathrm{reg}}}^{2}$, to partition the $(C T, A)$ space into configurations that promote regulatory $\mathrm{T}$ cell activation and those that do not. Generally, activated regulatory $\mathrm{T}$ cells migrate to the infection site when cytokine from effector $\mathrm{T}$ cells is high and antigen load is low, i.e. excessive immune inflammation.

\subsection{Macrophages}

Macrophages are scavengers that clear antigen/PAMP from the site of infection in a highly regulated operation. Regulation is achieved by careful management of the switch between the two macrophages states: resting (MA) and activated (MA) [2]. The partial differential equations characterizing these two macrophage states are shown below.

$$
\left\{\begin{array}{l}
\frac{\partial M R}{\partial t}=\overbrace{-\mu_{M R} M R}^{\text {natural }} \overbrace{-\gamma_{M R}\left(C T, C T_{r e g}, A\right) \cdot M R}^{\text {death }} \overbrace{+D_{M R} \Delta M R}^{\text {activation }} \underbrace{\text { effective diffusion } \overbrace{-\nabla\left(\chi_{M R}(M R \cdot \nabla C H)\right)}^{\text {effective chemotaxis }}}_{\text {activation }}, \\
\frac{\partial M A}{\partial t}=\underbrace{-\mu_{M A} M A}_{\text {degradation }} \underbrace{+\gamma_{M R}\left(C T, C T_{r e g}, A\right) \cdot M R}_{\text {diffusion }} \underbrace{+D_{M A} \Delta M A}_{\text {effective chemotaxis }} \underbrace{-\delta\left(\chi_{M A}(M A \cdot \nabla C H)\right)}_{\left.M R(\cdot, t)\right|_{\partial \Omega}=M R_{0},\left.\frac{\partial M A}{\partial n}\right|_{\partial \Omega}=0, M R(x, 0)=M R_{0}, M A(x, 0)=0 .}
\end{array}\right.
$$

Like all of the other immune cells in our model, macrophages are attracted to the source of antigen/PAMP by chemotaxis along a chemokine gradient established by neutrophils. In our model, resting macrophages patrol the tissue through effective diffusion, existing at some steady state level $M R_{0}$, representing the balance of macrophage recruitment and death in the tissues at rate $\mu_{M R}$. Initially, there are no activated macrophages. Then, as the discussion in the Section 2.4 indicates, effector and regulatory $\mathrm{T}$ cells compete to activate or block activation of resting macrophages with secreted cytokines [75]. These cytokine signals, along with antigen/PAMP levels, are sensed and processed by the macrophage [76], resulting in activation occurring at rate $\gamma_{M R}\left(C T, C T_{r e g}, A\right)$, where $C T$ and $C T_{\text {reg }}$ are the local concentrations of cytokine produced by effector and regulatory $\mathrm{T}$ cells, respectively, and $A$ is the local antigen/PAMP concentration. Knowing little about how these cytokine signals are actually interpreted, we model the activation rate as

$$
\gamma_{M R}\left(C T, C T_{r e g}, A\right)= \begin{cases}\gamma_{M R} & C T>\epsilon_{M A}^{1}+\epsilon_{M A}^{2} A, C T_{r e g}<\epsilon_{M A}^{3}+\epsilon_{M A}^{4} A, \\ 0 & \text { otherwise }\end{cases}
$$

which, similar to the $T_{\text {reg }}$ recruitment threshold function (6), partitions the $\left(C T, C T_{\text {reg }}, A\right)$ space such that macrophage activation occurs when inflammatory signal $C T$ dominates antigen/PAMP $A$ and antigen/PAMP, in turn, dominates suppressive signal $C T_{\text {reg. }}$. Upon activation, macrophages lose access to the microvasculature and are thus less mobile (diffusion rate $\left.D_{M A}<D_{M R}\right)$, but are voracious consumers of antigen/PAMP $\left(\lambda_{A \mid M A}>\right.$ $\lambda_{A \mid M R}$ ), and acquire additional skills, including the ability to phagocytize apoptotic 
neutrophils at rate $\lambda_{N D \mid M A}$ (see Equation 2 and Refs. [1,2,9]) and produce chemokine at rate $\beta_{C H \mid M A}$ (see Equation (9) and Ref. [53]).

\subsection{Chemical messengers: Chemokines and cytokines}

Chemokines are released by many different types of cells and serve to direct the movement of innate and adaptive immune cells [57,74]. Although there are many chemokines employed by the immune system, we utilize a single, generic chemokine to drive all forms of chemotaxis in our simple model. In our model, chemotaxis of all immune cells is determined by the concentration gradient of a generic chemokine $\mathrm{CH}$, produced by neutrophils at rate $\beta_{C H \mid N}$ and activated macrophages at rate $\beta_{C H \mid M A}$, in response to encounters of these cells with antigen/PAMP [63]. This inflammatory chemokine functions as a chemoattractant for neutrophils and other effector immune cells to the site of infection. The chemokine also decays at rate $\mu_{C H}$ and diffuses through the tissue with coefficient $D_{C H}$. The initial chemokine gradient is established by the rapid arrival of neutrophils to the source of antigen/PAMP through effective diffusion via the microvasculature.

$$
\left\{\begin{array}{l}
\frac{\partial C H}{\partial t}=\underbrace{\left(\beta_{C H \mid M A} M A+\beta_{C H \mid N} N\right) \cdot A}_{\text {secreted by } N \text { and } M A} \underbrace{-\mu_{C H} C H}_{\text {degradation }} \underbrace{+D_{C H} \Delta C H}_{\text {diffusion }}, \\
\left.\frac{\partial C H}{\partial n}\right|_{\partial \Omega}=0, C H(x, 0)=0 .
\end{array}\right.
$$

Cytokines are a family of proteins that are used for intercellular communication. They are important in both innate and adaptive immune responses. Cytokines are characterized by considerable 'redundancy,' in that many appear to share similar functions [27]. In our model, to avoid ambiguity and complexity of the roles of cytokines, we simplify the system with only three major cytokine categories: those produced by effector T cells, such as IL- 2 and IFN- $\gamma$, denoted by $C T$; cytokines produced by regulatory T cells, such as IL-10 and TGF- $\beta$, denoted by $C T_{\text {reg }}$; and cytokines produced by activated macrophages upon engulfing apoptotic neutrophils, such as IL-1 and TNF- $\alpha$, and denoted by $C M$ in the model [2] . $C T$ promotes regulatory $\mathrm{T}$ cell activation, a function of IL-2, and macrophage activation, a function of IFN- $\gamma . C T_{\text {reg }}$ regulates the pro-inflammatory immune response by blocking activation of macrophages. $C M$ is used to limit the recruitment of neutrophils.

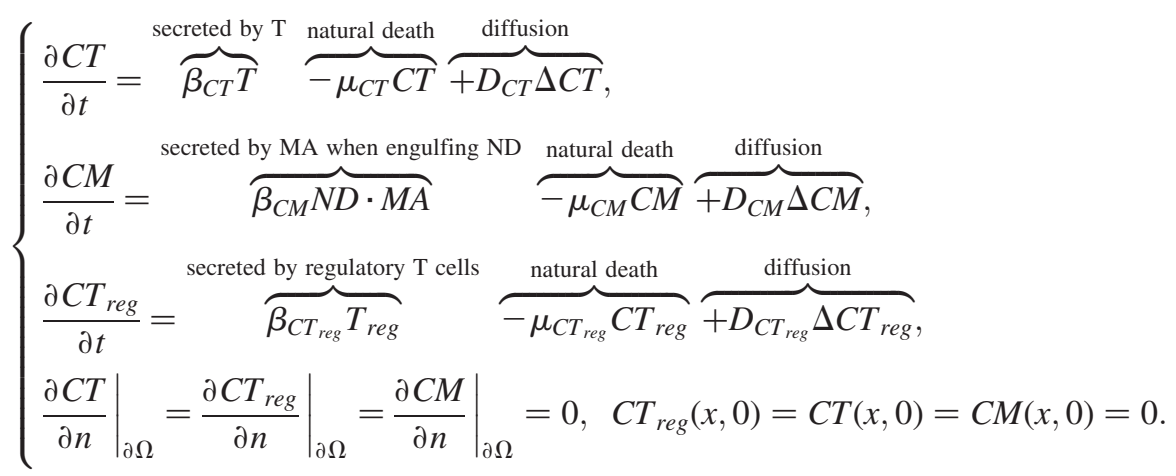




\section{Setting parameters}

To simulate and study the proposed PDE system, we must select values for all model parameters. Unfortunately, there are few direct estimates of most parameters, and in vivo immunological data may originate from multiple sources, for example mice or humans. We use whatever data is available, regardless of source, but acknowledge that substantial differences among species likely exist. Future calibration of model parameters against biological data will permit investigation of these differences. For parameters with no information, we have proposed what we think are reasonable values based on previously published immunological models and expert opinion. For discussion, we categorize the parameters of the system (1)-(10) into four groups: (1) initial conditions, (2) kinetic parameters, (3) diffusion and chemotaxis coefficients and (4) threshold function constants.

\subsection{Initial cell densities}

Because we have used mathematical homogenization to model vascularized tissue, any immune cell that normally flows through the blood stream exists at some initial level at the modelled site of infection. Other cells, such as mature dendritic cells or antigen specific $\mathrm{T}$ cells that are activated in response to the infection, and all cytokines, start at zero initial concentration everywhere and are not listed in Table 2.

There is data available on the abundance of immune cells in the blood. For simplicity, we assume the initial concentrations in the simulation area are equivalent to the concentrations in the blood. Neutrophils are the most abundant white blood cell in the vasculature, with a reported 2.5 to $7.5 \times 10^{9}$ cells per litre [7], so we choose the initial and boundary neutrophil concentration, $N_{0}$, in this range. Approximately $6 \%$ of white blood cells in humans are monocytes. In the tissue, most of these monocytes mature into macrophages [27], leading to $M R_{0} \sim 1 \times 10^{8} / \mathrm{L}$ [7]. A small fraction of monocytes become dendritic cells, with initial density in lymph node at $D E_{0} \sim 1 \times 10^{7} / \mathrm{L}[11]$.

\subsection{Kinetic parameters}

We further classify the kinetic parameters into three classes: death/decay rates, production and uptake rates, and regulation rates, where the rates of regulatory processes often involve cell-to-cell contact.

\subsubsection{Death rates of cells and decay rates of cytokines}

Apparently one of the easier quantities to measure experimentally, there is abundant data on the lifespan of immune cells as recorded in Table 3. Here, the evidence shows that neutrophils are short-lived [49], unlike macrophages, which live for months [58]. Activated or mature immune cells tend to live shorter lives than their unactivated/ immature cousins $[37,52,53]$. According to [47], it is estimated that $3 \%$ of $\mathrm{T}$ cells are lost

Table 2. Initial cell densities.

\begin{tabular}{llccc}
\hline Name & Cell type & Biological range & Simulation value & Reference \\
\hline$N_{0}$ & Neutrophil & {$[2.5,7.5] \times 10^{9} / L$} & $2.5 \times 10^{9}$ & {$[7]$} \\
$D E_{0}$ & Immature dendritic cell & & $5 \times 10^{7}$ & {$[11]$} \\
$M R_{0}$ & Resting macrophage & $\sim 1 \times 10^{8} / L$ & $1.5 \times 10^{8}$ & {$[7]$} \\
\hline
\end{tabular}


Table 3. Death rates of cells and decay rates of proteins (unit/day).

\begin{tabular}{|c|c|c|c|c|}
\hline & Rate of ... & Biological range & Simulation & Reference \\
\hline Name & ... Death & & & \\
\hline$\mu_{N}$ & Neutrophil apoptosis & $0.69-2.08 /$ day & 1.39 & {$[24,42,60]$} \\
\hline$\mu_{D E}$ & Immature dendritic cell death & $0.23-0.35 /$ day & 0.25 & [37] \\
\hline$\mu_{D A}$ & Mature dendritic cell death & $0.69-1.39 /$ day & 1.00 & [37] \\
\hline$\mu_{T}$ & Effector $\mathrm{T}$ cell death & $0.03-0.333 /$ day & 0.33 & {$[33,47,66]$} \\
\hline$\mu_{T_{\text {reg }}}$ & Regulatory $\mathrm{T}$ cell death & $0.03-0.333 /$ day & 0.33 & [33], estimate \\
\hline$\mu_{M R}$ & Resting macrophage death & $0.0033-0.007 /$ day & 0.0033 & {$[52,53]$} \\
\hline \multirow[t]{2}{*}{$\mu_{M A}$} & Activated macrophage death & $0.07 /$ day & 0.07 & {$[52,53]$} \\
\hline & ... Decay & & & \\
\hline$\mu_{N D}$ & Apoptotic neutrophil decay & & 0.05 & Estimate \\
\hline$\mu_{A}$ & Antigen/PAMP decay & $\sim 10^{-4} /$ day & 0.005 & Estimate \\
\hline$\mu_{C H}$ & Chemokine decay & & 14.4 & Estimate \\
\hline$\mu_{C T}$ & $\mathrm{~T}$ cell cytokine decay & 2.16-33.2/day & 2.16 & {$[13,21]$} \\
\hline$\mu_{C T_{\text {reg }}}$ & $\begin{array}{l}\text { Regulatory T cell } \\
\text { Cytokine decay }\end{array}$ & $3.6969-7.23 /$ day & 3.70 & [76] \\
\hline$\mu_{C M}$ & $\begin{array}{l}\text { Activated macrophage } \\
\text { Cytokine decay }\end{array}$ & 2.16/day & 2.16 & {$[2,67]$} \\
\hline
\end{tabular}

per day, so we estimate $\mu_{T}=-\ln (1-0.03) \approx 0.03 /$ day. The half-life of neutrophils is reportedly $8-24 \mathrm{~h}[24,42,60]$, yielding the range of Table 3 . With no information for regulatory $\mathrm{T}$ cells, we assume effector and regulatory $\mathrm{T}$ cells share similar death rates.

The other entities in the model are not living cells, and most have high decay rates typical of proteins. Apoptotic neutrophils, however, are large objects, cleared through phagocytosis by macrophages, not through decay, so we assign them a small decay rate. Antigen/PAMP, represent a pathogen that actively avoids its own decay, so we have also assigned $\mu_{A}$ to be small.

\subsubsection{Production and uptake rates}

There are data available on the rates of chemokine/cytokine production by immune cells, however, since the chemical messengers of our model perform the functions of multiple biological molecules, our secretion rates, reported in Table 4, can only approximate true secretion rates. While in our model there is only one chemokine, neutrophils and activated macrophages secrete this chemokine at distinct rates $\beta_{C H \mid N}<\beta_{C H \mid M A}$. The three cytokines $C T, C T_{\text {reg }}$ and $C M$ are secreted by effector $\mathrm{T}$ cells, regulatory $\mathrm{T}$ cells, and activated macrophages, respectively.

The processing (uptake/phagocytosis/pinocytosis) rates for antigen/PAMP in Equation (1) vary by immune cell. Activated macrophages are three times more efficient antigen processors than resting macrophages [56], but immature dendritic cells are the most efficient antigen processors of all [61]. Again, we expect most antigen/PAMP to be consumed by phagocytic cells, so the decay rate $\mu_{A}$ is a lower bound on processing rates. These claims yield

$$
\lambda_{A \mid D E}>\lambda_{A \mid M A} \lambda_{A \mid N}>\lambda_{A \mid M R}
$$


Table 4. Production and uptake rates.

\begin{tabular}{|c|c|c|c|c|}
\hline Symbol & Rate of ... & Biological range & Simulation & Reference \\
\hline & Secretion of ... & & & \\
\hline$\beta_{C H \mid M A}$ & $\begin{array}{l}\text { Chemokine by } \\
\text { activated macrophage }\end{array}$ & $0.3-15.0 \times 10^{-3} /$ cell $/$ day & 0.1 & {$[3]$} \\
\hline$\beta_{C H \mid N}$ & $\begin{array}{l}\text { Chemokine by } \\
\text { neutrophil }\end{array}$ & $\sim \beta_{C H \mid M A}$ & 0.4 & Estimate $^{\mathrm{a}}$ \\
\hline$\beta_{C T}$ & $\begin{array}{l}\text { cytokine by effector } \\
\mathrm{T} \text { cell }\end{array}$ & $0.02-0.066 \mathrm{pg} / \mathrm{cell} / \mathrm{day}$ & 0.06 & {$[20]$} \\
\hline$\beta_{C T_{\text {reg }}}$ & $\begin{array}{l}\text { Cytokine by regulatory } \\
\mathrm{T} \text { cell }\end{array}$ & $2-60 \times 10^{-4} \mathrm{pg} / \mathrm{cell} /$ day & 0.06 & Estimate, $[76]^{\mathrm{a}}$ \\
\hline \multirow[t]{2}{*}{$\beta_{C M}$} & $\begin{array}{l}\text { Cytokine by activated } \\
\text { macrophage }\end{array}$ & & 3 & Estimate, $[2,6]$ \\
\hline & Uptake of ... & & (unit:/cell/day) & \\
\hline$\lambda_{A \mid M A}$ & $\begin{array}{l}\text { Antigen/PAMP by } \\
\text { activated macrophage }\end{array}$ & $10^{-7}$ to $10^{-5}$ & 0.8 & {$[18,52,53]$} \\
\hline$\lambda_{A \mid M R}$ & $\begin{array}{l}\text { Antigen/PAMP by } \\
\text { resting macrophage }\end{array}$ & b & 0.25 & {$[56]$} \\
\hline$\lambda_{A \mid N}$ & $\begin{array}{l}\text { Antigen/PAMP by } \\
\text { neutrophil }\end{array}$ & b & 0.55 & \\
\hline$\lambda_{A \mid D E}$ & $\begin{array}{l}\text { Antigen/PAMP by } \\
\text { immature dendritic cell }\end{array}$ & $\mathrm{b}$ & 1.50 & Estimate, [61] \\
\hline$\lambda_{N D \mid M A}$ & $\begin{array}{l}\text { Apoptotic neutrophil } \\
\text { by activated } \\
\text { macrophage }\end{array}$ & b & 2.60 & \\
\hline$\lambda_{N \mid A}$ & Degranulation rate & b & 0.55 & \\
\hline
\end{tabular}

${ }^{a}$ Values that are model specific.

${ }^{\mathrm{b}}$ See in context.

Concrete data suggests antigen processing rates for activated macrophage are in the range $10^{-7}$ to $10^{-5}$ per second $[18,52,53]$. We set, in our units, $\lambda_{\mathrm{A} \mid \mathrm{MA}}=0.8 / \mathrm{cell} / \mathrm{day}$, and select other processing rates to satisfy (11).

\subsubsection{Regulation rates}

Very little is known about immune self-regulation, so the functions and parameters of Table 5 are our simple proposals. It is known that neutrophils invading the site of an infection produce auto-inhibitory factors [54,77]. Since neutrophils produce the vast majority of infection site chemokine early in infection, we let the concentration of chemokine stand in for all such factors. Then, $\delta_{\mathrm{N} \mid \mathrm{CH}}$ is the rate of neutrophil degradation due to this feedback loop.

The maturation of immature dendritic cells, activation of resting macrophages, and the recruitment of regulatory $\mathrm{T}$ cells by activation in the lymph nodes are threshold functions that we have previously defined in Sections 2.3 and 2.4. Reported in Table 5 are the maximum activation rates. There is further discussion about when maximal rates are achieved in the following Section 3.3.

\subsection{Threshold functions and constants}

Most immune actions are tightly regulated, such that they are promoted in certain environments and blocked in others. Immune actions typically exist on a continuous scale, 
Table 5. Regulation rates.

\begin{tabular}{llccc}
\hline Symbol & Rate of... & Biological range & Simulation & Reference \\
\hline$\delta_{N \mid C H}$ & Auto-regulation of neutrophil & & 0.3 & Estimate $^{\mathrm{b}}$ \\
$\delta_{T \mid T_{\text {reg }}}$ & Downregulation of effector T cells & $0.05-0.5 /$ cell/day & 0.3 & {$[33]$} \\
$\gamma_{D E}{ }^{\mathrm{c}}$ & Dendritic cell maturation & $0.01-0.65 /$ day & 0.4 & {$[31,33]$} \\
$\gamma_{M R}{ }^{\mathrm{c}}$ & Resting macrophage activation & $0.2-0.4 /$ day & 0.4 & {$[76]$} \\
$\gamma_{T \mid D A}$ & Effector T cell recruitment & \\
$\gamma_{T_{\text {reg }}}$ & Regulatory T cell recruitment & $\sim \gamma_{T \mid D A}$ & 2.5 & Estimate $^{\mathrm{a}}$ \\
\hline
\end{tabular}

${ }^{\mathrm{a}} \mathrm{Unit} / \mathrm{CH} /$ day.

${ }^{\mathrm{b}}$ Values that are model specific.

${ }^{\mathrm{c}}$ Maximum rates.

${ }^{\mathrm{d}}$ Unit/cell/day.

for example phagocytosis can occur at a continuum of rates. Cytokines, other molecular signals, and even cells in the environment are sensed via cell surface receptors, and processed via signal transduction networks inside the cell to output the cellular activity level. One can think of cells and their networks as functions that map environmental states to cellular actions. In most cases, the explicit expressions of the functions are unknown. In our simple model, we assume these functions are step functions that map the relevant environmental quantities to an on/off state of activity. As a consequence, we specify two activity levels: maximal on and off. We also assume that the space of environmental conditions can be simply partitioned between those environments that turn activity on and those that turn it off. Immune activities that are modelled in this way are neutrophil recruitment, dendritic cell maturation, regulatory $\mathrm{T}$ cell recruitment, and macrophage activation (Table 6).

\subsection{Diffusion and chemotaxis rates}

The chemotaxis and diffusion rates for immune cells in Table 7 are larger than those measured in vitro because the circulation of immune cells in the microvasculature, governed by Stoke's equation, has been approximated by effective chemotaxis and diffusion $[12,68,70]$, as discussed in Section 2.2. Apoptotic neutrophils created in the extravascular tissue do not re-enter the vascular space. They diffuse at real rate $0.0001 \mu \mathrm{m}^{2} / \mathrm{min}$, substantially slower than proteins like antigen/PAMP, chemokines and cytokines. Finally, we have assumed that antigen/PAMP, representing pathogen, is localized at the infection site, corresponding to relatively small diffusion of $A$.

Table 6. Threshold parameters.

\begin{tabular}{llcl}
\hline Name & Description & Simulation & Reference $^{\text {a }}$ \\
\hline$\epsilon_{N}$ & Neutrophil recruitment & 0.2 & Estimate $^{\mathrm{a}}$ \\
$\epsilon_{D E}$ & Dendritic cell maturation & $1.0 \times 10^{-5}$ & Estimate $^{\mathrm{a}}$ \\
$\epsilon_{T_{\text {reg }}}^{1}$ & Regulatory T cell recruitment & 0.001 & Estimate $^{\mathrm{a}}$ \\
$\epsilon_{T_{\text {reg }}}^{2}$ & & 0.05 & Estimate $^{\mathrm{a}}$ \\
$\epsilon_{M A}^{1}$ & Macrophage activation & 0.001 & Estimate $^{\mathrm{a}}$ \\
$\epsilon_{M A}^{2}$ & & 0.05 & Estimate $^{\mathrm{a}}$ \\
$\epsilon_{M A}^{3}$ & & 0.001 & Estimate $^{\mathrm{a}}$ \\
$\epsilon_{M A}^{4}$ & & -0.05 & Estimate $^{\mathrm{a}}$ \\
\hline
\end{tabular}

${ }^{\mathrm{a}}$ Values that are model specific. 
Table 7. Chemotaxis and diffusion rates.

\begin{tabular}{|c|c|c|c|c|}
\hline Name & Cell & Biological range & Simulation & Reference \\
\hline \multicolumn{5}{|c|}{ Chemotaxis rate $\left(\times 10^{-10} \mathrm{M}\right)$} \\
\hline $\begin{array}{l}\chi_{N} \\
\chi_{D E} \\
\chi_{D A} \\
\chi_{T} \\
\chi_{T_{\text {reg }}} \\
\chi_{M R} \\
\chi_{M A}\end{array}$ & $\begin{array}{l}\text { Neutrophils } \\
\text { Immature dendritic cells } \\
\text { Mature dendritic cells } \\
\text { Effector T cells } \\
\text { Regulatory T cells } \\
\text { Resting macrophages } \\
\text { Activated macrophages }\end{array}$ & $\begin{array}{c}10-13.3 \mu \mathrm{m}^{2} / \mathrm{min} \\
3.5-11 \mu \mathrm{m}^{2} / \mathrm{min} \\
\sim \chi_{D E}, \mathrm{smaller}^{2} \\
2-10 \mu \mathrm{m}^{2} / \mathrm{min} \\
\sim \chi_{T} \\
\sim \chi_{M A} \\
3.11-12.95 \mu \mathrm{m}^{2} / \mathrm{min}\end{array}$ & $\begin{array}{l}10 \\
4.5 \\
3.5 \\
6.5 \\
6.5 \\
2.5 \\
3.0\end{array}$ & $\begin{array}{l}\text { Estimate }^{\mathrm{a}}[46] \\
\text { Estimate }^{\mathrm{a}} \\
\text { Estimate }^{\mathrm{a}} \\
\text { Estimate }^{\mathrm{a}}[51] \\
\text { Estimate }^{\mathrm{a}} \\
\text { Estimate }^{\mathrm{a}} \\
{[35,65,72]}\end{array}$ \\
\hline \multicolumn{5}{|c|}{ Diffusion rate } \\
\hline$D_{A}$ & Antigen/PAMP & $0.005 \mu \mathrm{m}^{2} / \mathrm{min}$ & 0.005 & Estimate $^{\mathrm{b}}$ \\
\hline$D_{C H}$ & Chemokine & $0.6-60 \mu \mathrm{m}^{2} / \mathrm{min}$ & 6.4 & {$[18,53]$} \\
\hline$D_{N}$ & Neutrophil & $8-11 \mu \mathrm{m}^{2} / \mathrm{min}$ & 10 & Estimate $^{\mathrm{a}}[16]$ \\
\hline$D_{N D}$ & Apoptotic neutrophil & $0.0001 \mu \mathrm{m}^{2} / \mathrm{min}$ & 0.0001 & Estimate \\
\hline$D_{D E}$ & Immature dendritic cell & $\sim D_{D A}$ & 4.5 & Estimate $^{\mathrm{a}}$ \\
\hline$D_{D A}$ & Mature dendritic cell & $4-6.6 \mu \mathrm{m}^{2} / \mathrm{min}$ & 4.5 & Estimate $^{\mathrm{a}}[44,45]$ \\
\hline$D_{T}$ & Effector $\mathrm{T}$ cell & $6-11 \mu \mathrm{m}^{2} / \mathrm{min}$ & 6.0 & Estimate $^{\mathrm{a}}[48]$ \\
\hline$D_{\text {Treg }}$ & Regulatory $\mathrm{T}$ cell & $\sim D_{T}$ & 6.0 & Estimate $^{\mathrm{a}}$ \\
\hline$D_{M R}$ & Resting macrophage & $\sim D_{M R}$ & 3.0 & Estimate $^{\mathrm{a}}$ \\
\hline$D_{M A}$ & Activated macrophage & $6 \times 10^{-2}-6 \mu \mathrm{m}^{2} / \mathrm{min}$ & 3.0 & Estimate $^{\mathrm{a}} *[18,38,52]$ \\
\hline$D_{C T}$ & $\begin{array}{l}\text { Effector T cell-released } \\
\text { cytokine }\end{array}$ & $6.4-1.5 \times 10^{2} \mu \mathrm{m}^{2} / \min$ & 6.4 & Estimate $[46,52]$ \\
\hline$D_{C M}$ & $\begin{array}{l}\text { Macrophage-released } \\
\text { cytokine }\end{array}$ & $\sim D_{C H}$ & 6.4 & Estimate [38] \\
\hline$D_{C T_{\text {reg }}}$ & $\begin{array}{l}\text { Regulatory } \mathrm{T} \text { cell-released } \\
\text { cytokine }\end{array}$ & $\sim D_{C T}$ & 6.4 & Estimate \\
\hline
\end{tabular}

${ }^{\mathrm{a} B y}$ homogenization $[68,70]$.

${ }^{\mathrm{b}}$ Values that are model specific.

\section{Introduction of numerical simulations}

In this section, we will make some necessary preparations for the numerical experiments by discussing the model boundary conditions in Section 4.1 and outlining the numerical methods [39] used for simulation in Section 4.2.

\subsection{Boundary conditions}

In the PDE system (1)-(10), there are 13 variables listed in Table 1. Centred at the origin, the domain for the PDE system is assumed to be a bounded open connected set, which we denote by $\Omega$. The boundary of $\Omega$, denoted by $\partial \Omega$, represents the lymph nodes.

The initial distribution of antigen, $A(x, 0)$, is determined by the source function $g(x, 0)$, which is zero for $|x| \geq r$. In all cases, the radius of antigen source is substantially smaller than the simulation area. Combined with the small diffusion rate of antigen, we expect little antigen to reach the lymph nodes, so the boundary value of antigen is set to be zero, $\left.A(\cdot, t)\right|_{\partial \Omega}=0$.

Since we model the immune response in a local region, the total exchange of cytokines through the boundary should be balanced, which implies $\vec{n} \cdot \nabla U=0$ for any protein $U$. 
Hence, we set Neumann boundary conditions for the cytokines, i.e

$$
\left.\frac{\partial U}{\partial \vec{n}}\right|_{\partial \Omega}=0, \text { where } U=C T, C M, C T_{r e g}, C H
$$

Likewise, we assume balanced flux of apoptotic neutrophils, mature dendritic cells, and activated macrophages on the boundary of the domain, so

$$
\left.\frac{\partial V}{\partial \vec{n}}\right|_{\partial \Omega}=0, \text { where } V=N D, D A, M A .
$$

The boundary conditions for other immune cells are constant, reflecting a steady state source (resting macrophages and immature dendritic cells), or state-dependent to emulate activation and recruitment at the lymph nodes (effector and regulatory $\mathrm{T}$ cells). More discussion of recruitment boundary conditions can be found in Sections 2.4 and 3.3.

\subsection{Numerical method}

The fundamental equation of the nonlinear PDE model (1)-(10) is the Keller-Segel equation modelling chemotaxis [32].

$$
\left\{\begin{array}{l}
\rho_{t}-\operatorname{div}(\chi(\nabla c \cdot \rho))=\mu \Delta \rho \\
c_{t}-\Delta c=\alpha \rho-\beta c \\
\frac{\partial \rho}{\partial \vec{n}}=\frac{\partial c}{\partial \vec{n}}=0 \quad x \in \partial \Omega
\end{array}\right.
$$

Equations (1), (9) and (10) are reaction diffusion equations for antigen/PAMP, chemokine, and cytokines. The equations involving the dynamics of immune cells, (2), (4), (5) and (7), are chemotaxis equations where the convection term $\operatorname{div}\left(\chi_{U}(\nabla C H \cdot U)\right)$ contributes to the chemoattraction effect.

Numerical computation of chemotaxis equations is a difficult mathematical task. Different numerical approaches to simulate Equation (14) or similar systems have been proposed in $[17,38,39,40]$. Two issues must be addressed for the numerical simulation of this model: resolution and computing time. To avoid misleading information, high resolution is required. Implicit and conservative schemes are used for approximation. Particularly, combining a backward scheme with time, we can derive an accurate approximation of the convection equations by a central scheme and an upwind scheme [38,39]. More importantly, the schemes we apply for simulation are unconditionally stable and do not require small time steps. For the simulation of heat equations, implicit methods are applied to the reaction diffusion Equations (1), (9) and (10). These implicit methods give $o(h)$ accuracy for both spatial and time directions and avoid potential oscillation for solutions, thus it guarantees the stability of our approximation [38,39,71].

The computing time of the system of PDEs is another important concern for our choice of numerical schemes. With a small time step, an explicit scheme can yield the same resolution as the implicit scheme. However, the simulation time with the explicit scheme is intolerable. In addition, explicit schemes are not stable, which causes a unstable simulation after some finite steps. In our simulation, the time step size is determined mainly by the convection equations, since the implicit schemes we use to approximate the diffusion equations are unconditionally stable. We use CFL conditions to determine 
the time step size, but the diffusion terms in those chemotaxis equations suggest the use of a modified version of the traditional one [38,39,71]. According to [38,39], since the flux is bounded in the numerical domain, in the simulation we use the following CFL condition

$$
\Delta t=\min _{U \in V}\left\{\alpha \Delta x^{2}\left(D_{U}+\frac{\left|\nabla C H \cdot \chi_{U}\right| \Delta x}{2}\right)^{-1}\right\}
$$

where $0<\alpha<1$ and $V=\left\{N, T_{\text {reg }}, T, D E, D A, M A, M R\right\}$ is a set of variables.

\section{Numerical results}

In this section, we examine the model by conducting a series of numerical experiments. In Section 5.1, we show that the model in (1)-(10) contains the necessary immunological terms to recapitulate the immune stages of initiation, effector response, and resolution or equilibration. The numerical domain for all simulations in the one-dimensional case is the interval $[-2,2]$, and a rectangle $[-2,2] \times[-2,2]$ in two-dimensional case. All parameters, initial conditions, and boundary conditions are the same in these simulations. In Section 5.2, we demonstrate the dynamics of the various cell populations in both one- and twodimensionals as the model responds to both acute and chronic infection. In Section 5.3, we use a two-dimensional simulation to demonstrate how the model forms a persistent granuloma in response to chronic infection, whereas in the acute case the granuloma is diminished and finally eliminated.

\subsection{Minimal set of immune factors}

One of our main objectives is to identify a minimal set of cells, chemical messengers, and interactions to recapitulate a functional immune system. A guiding principle is that the immune system should be robust yet tolerant of persistent infections. Furthermore, hosts should incur minimal damage, as measured by levels of inflammatory cells and cytokines, from persistent infections. It is well known that both macrophages and effector $\mathrm{T}$ cells play primary roles in an effective immune response [27], thus we focus our interest on exploring the roles of the dendritic cells and regulatory $\mathrm{T}$ cells.

For simplicity, we assume the antigen/PAMP (source) appears at the origin in a radially symmetric region with radius $r$. Here, we will explore source functions $g(x, t)$ of the form

$$
g(x, t)= \begin{cases}g_{0} \mathrm{e}^{-g_{1} t} & |x|<r \\ 0, & \text { otherwise }\end{cases}
$$

where both $g_{0}$ and $g_{1}$ are non-negative constants. We distinguish chronic and acute antigen exposures by the value of $g_{1}$. If $g_{1}=0$ the antigen/PAMP source is persistent, as might be the case for a resistant, replicating pathogen; otherwise, if $g_{1}>0$ the source of antigen is transient, representing an acute infection.

In our first experiment, we deliver persistent antigen in the centre of the onedimensional domain by letting $g_{1}=0, g_{0}=8$, and $r=0.8$ in Equation (16). For the simulation, we partition the interval $\Omega=[-2,2]$ into $n=40$ subintervals with spatial step size $\Delta x=1 / 10$. Under this scenario, we investigate the temporal dynamics of the local average mass of macrophages (both resting and active), $\mathrm{T}$ cells (both effector and 
regulatory), neutrophils and antigen/PAMP. The local average mass is defined as

$$
U_{\text {local }}(t)=\frac{1}{\left|\Omega_{0}\right|} \int_{\Omega_{0}} U(x, t) \mathrm{d} x,
$$

where $\Omega_{0} \subseteq \Omega$ is the local infection site defined as

$$
\Omega_{0}=\left\{x \in R^{1}:|x| \leq r_{0}\right\} \subseteq \Omega,
$$

and $U \in\left\{(M A+M R),\left(T+T_{\text {reg }}\right), N, C H\right\}$. In the following simulation, we use $r_{0}=0.5$. The total mass of antigen, or antigen load, is defined as

$$
A_{\text {total }}(t)=\int_{\Omega} A(x, t) \mathrm{d} x .
$$

In Figure 4, we plot the local average mass of neutrophils, macrophages, and T cells as well as antigen load over time for the full model defined by Equations (1)-(10) and various reduced models. Also, the tan dash-dot lines in the figure represent the immune response under the full model during an acute infection, where $g_{1}=10$ and other conditions are as in the persistent antigen case. The full model under persistent infection (red lines) demonstrates an early and short burst of neutrophil activity at the infection site. By 10 days after antigen delivery, there are effectively no neutrophils left. The next cell type to dominate the infection site are the macrophages, which arrive before $\mathrm{T}$ cells and peak five days after antigen delivery. T cell numbers peak at seven days. The cell arrival sequences agree with that observed during in vivo granuloma formation [64]. Macrophages, T cells, and antigen load reach new steady state levels, also consistent with stable granuloma formation. The modelled granuloma is dominated by macrophages, which outnumber $\mathrm{T}$ cells by about 10:1, in agreement with in vivo observations $[7,49]$.
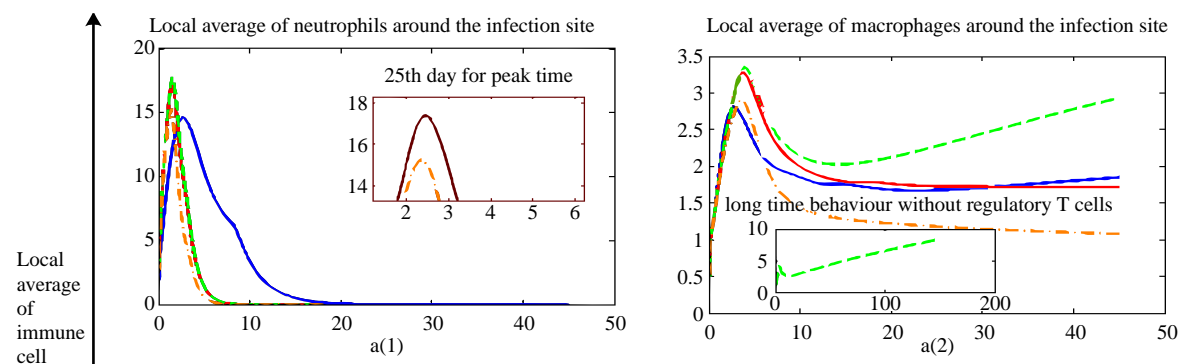

cell Local average of $\mathrm{T}$ cells around the infection site

and
total
mass

mass
of

antigen
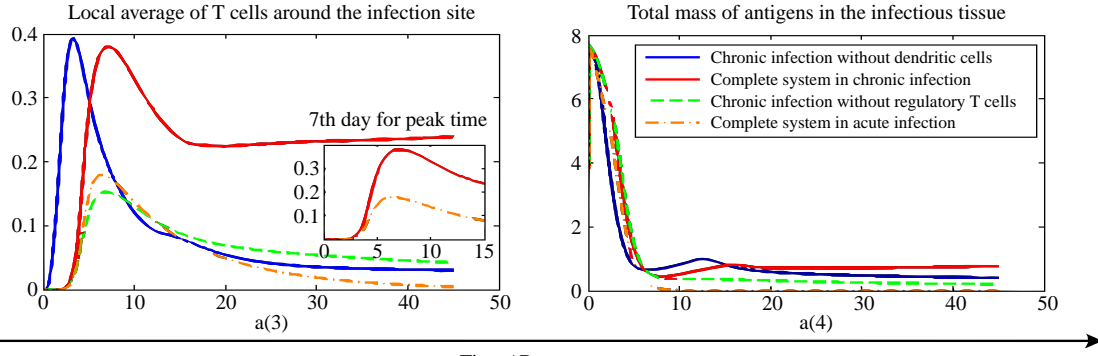

Time / Days

Figure 4. Plots a(1) to a(4) characterize the dynamics of the local mass for neutrophils, macrophages and $\mathrm{T}$ cells (including regulatory $\mathrm{T}$ cells) around the infection site in a chronic infection. Embedded plots in a(1) and a(3) are highlights of peak time for neutrophils and T cells. 
The newly equilibrated immune response controls antigen load 120 times lower than steady state conditions in the absence of an immune response. This outcome contrasts with acute infection, where the system approaches the initial, resting state a few days after antigen levels draw nigh on zero. T cells are the slowest to disappear, likely a consequence of our failure to include an active $\mathrm{T}$ cell downregulation mechanism after the antigen threat has vanished (Figure 4).

To test the role of regulatory $\mathrm{T}$ cells, we remove them from the model. The dashed green lines of Figure 4 display the results. Early macrophage recruitment and behaviour is similar to the full model, but instead of approaching a new equilibrium, macrophage numbers increase over time. Even after 150 days, the macrophage count is still increasing (see inset plot of a(2)). This increase continues despite very low antigen loads, less than $10 \%$ of the starting conditions. The plot of antigen load over time shows that antigen clearance is efficient early in infection, but becomes increasingly inefficient, requiring more and more macrophages, as antigen levels drop. As expected, the total number of $\mathrm{T}$ cells at the infection site is lower because regulatory $\mathrm{T}$ cells are not included.

We next test the role of dendritic cells in the model. We eliminate the terms for dendritic cells and set the boundary value of effector $\mathrm{T}$ cells proportional to the product of total antigen mass and total macrophage mass. We justify this boundary condition by noting that the more antigen and the more phagocytes available to transport the antigen to the lymph nodes, the more effector $\mathrm{T}$ cells will be primed and recruited. The results of this reduced model are shown as blue solid lines in Figure 4. The most important consequence of this change is the timing of macrophage and $\mathrm{T}$ cell recruitment to the site of infection. These two cell types now arrive simultaneously, in disagreement with the known temporal order for immune cell recruitment to granuloma [64]. The role of dendritic cells to transport antigen to the lymph nodes is a critical part of $\mathrm{T}$ cell recruitment, but takes time and necessarily delays $\mathrm{T}$ cell arrival at the infection site. Failure to model this delay, as we have done explicitly with dendritic cells, can lead to unrealistic behaviour and structure at the granuloma. Moreover, the total amount of T cells is only $2 \%$ of the macrophages when they approach equilibrium, for which is lower than previously described biological system [7,49].

With these three experiments, we conclude our modelled immune system constitutes a minimal set of immune factors that recapitulate a robust immune response, giving the dynamic behaviour of both acute and chronic infections.

\subsection{Spatial and temporal dynamics of immune system}

\subsubsection{One-dimensional experiments}

We now examine the spatio-temporal distribution of immune cells during chronic infection. We use the same experiment involving the full model from the previous section.

Figure 5 shows the spatio-temporal evolution of antigen, macrophage and chemokine concentrations in tissue during a chronic infection. Over time, the antigen density decreases due to uptake and processing by immune cells (Figure $5 \mathrm{e}(1)$ ). Initially, antigen concentration is markedly concave at the centre of the symmetric infection area, where immune cell density peaks. Over time, a new steady state is reached where antigen levels are low and nearly constant over the source area. The density of macrophages increases at the infection site in the early stage of the immune response, but then experiences a small decrease when antigen loads fall very low (Figure $5 \mathrm{e}(2))$. The immediate influx of neutrophils leads to a rapid increase in the chemokine concentration at the infection site (Figure $5 \mathrm{e}(3)$ ). The resulting gradient persists throughout infection even as macrophages take over the role of chemokine production, but the total mass of chemokine decreases to a new steady state. 


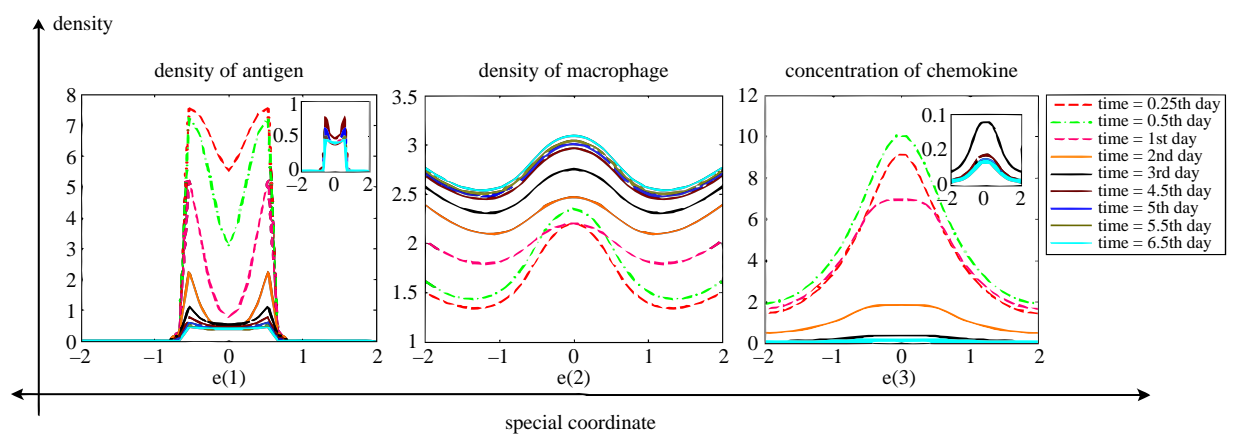

Figure 5. Plots e (1) to e(3) characterize the dynamics of antigens, macrophages and chemokines in spatial-temporal space in response to a chronic infection. Subplots in e(1) and e(3) illustrate the later stage (up to 6.5th day).

Figure 6 plots the spatio-temporal dynamics of effector and regulatory T cells. It is clear that both effector $\mathrm{T}$ cells and regulatory $\mathrm{T}$ cells are widely distributed in the tissue and directed to the site of infection gradually. It is worthwhile to point out that regulatory $\mathrm{T}$ cells and effector $\mathrm{T}$ cells exists at the infection site in near equal quantity.

Finally, the immune response in chronic and acute infections are compared in Figure 7. Figure 7 shows that macrophages dominate the infection response in the late stage without neutrophils, and total $\mathrm{T}$ cells are about $15 \%$ of macrophage, which is consistent with the known inflammatory response [49].

\subsubsection{Two-dimensional experiment}

In the 2D simulation, initial antigen load is

$$
A(x, 0)= \begin{cases}8, & |x|^{2}=x_{1}^{2}+x_{2}^{2}=r^{2} \leq 0.8 \\ 0, & \text { otherwise }\end{cases}
$$

All of the parameters are identical to the one-dimensional simulations, but we only consider chronic infection here. Generally, the results are consistent between one
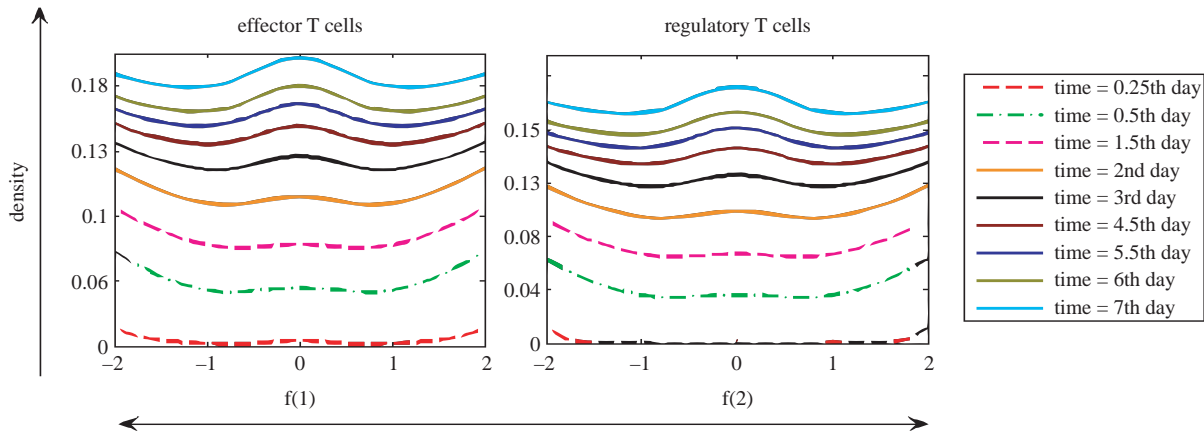

spatial coordinate

Figure 6. Plots $\mathrm{f}(1)$ and $\mathrm{f}(2)$ characterize the dynamics of effector and regulatory $\mathrm{T}$ cells in spatial-temporal space in response to a chronic infection. 

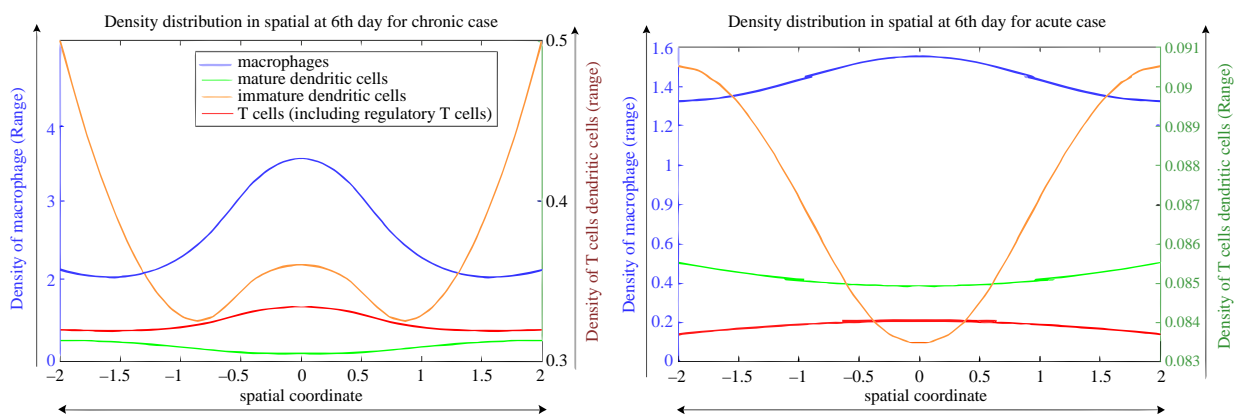

Figure 7. Density distribution of T cells, macrophages, immature and mature dendritic cells in response to chronic and acute infection on the 6th day, respectively. Note the different density scales between macrophages and other immune cells: the left $y$-axis refers to the macrophages while the right $y$-axis refers to all other immune cells.

and two dimensions. Here, we define the antigen/PAMP source domain to be $\Omega_{0}=\left\{x \in R^{2}|| x \mid \leq 1 / 2\right\}$.

A 'pseudo-oscillation' of chemokine can be observed in Figure 8, resulting when macrophages take over the role of neutrophils in producing chemokine. Figure 9 shows the dynamics of activated macrophages. As time progresses, density of activated macrophages increases but becomes increasingly localized at the Antigen/PAMP persistent source. More interestingly, we observe an oscillation of the local mass of activated macrophages. The appearance of this oscillation is due to the balance between macrophage activation

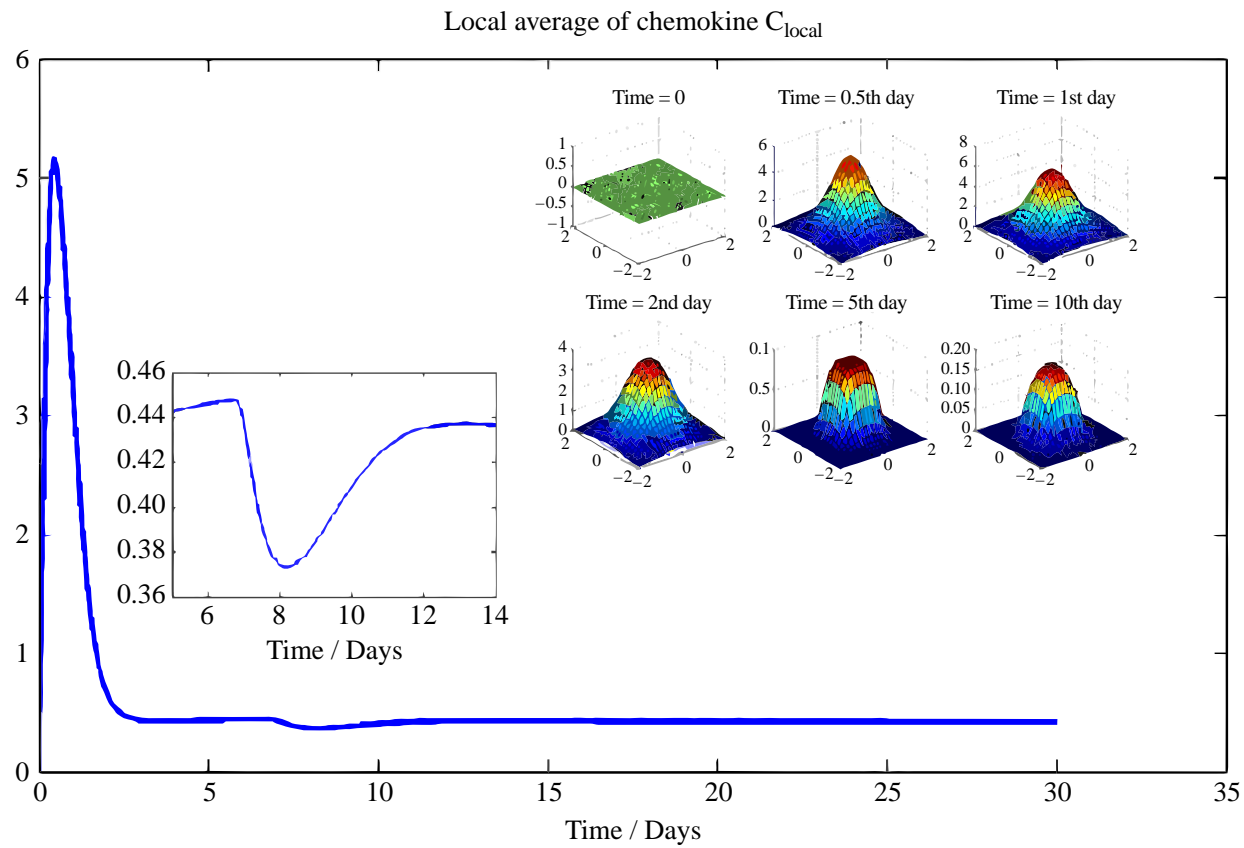

Figure 8. Dynamics of local average mass of chemokine with respect to time. Pseudo-oscillation occurs around the 8th day, as shown in embedded plot from days 4 to 14. Also embedded are threedimensional plots of the concentrations of chemokines across the simulation domain at several timepoints. 


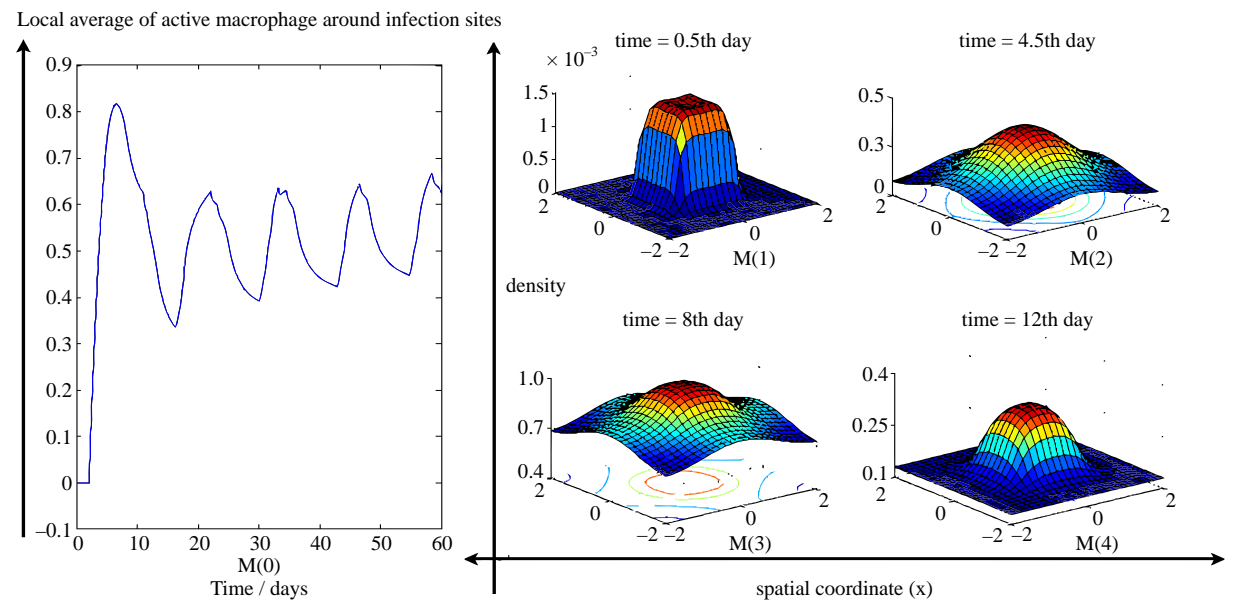

Figure 9. Plots $\mathrm{M}(0)$ to $\mathrm{M}(4)$ characterize the evolution of the density of activated macrophage in spatial-temporal space in response to a chronic infection. Note the density of active macrophages oscillates around the steady state.

and downregulation via the action of the effector and regulatory $\mathrm{T}$ cell secreted cytokines ( $C T$ and $C T_{\text {reg }}$ in the model).

Figure 10 demonstrates the dynamics of dendritic cell density. From r(1) to $r(3)$, the mature dendritic cell density starts highest at the site of infection and then gradually moves away towards the boundary (lymph nodes), which implies there is a spatially defined time delay. In $r(4)-r(6)$ the density of immature dendritic cells is highest at the boundary (lymph nodes) and the immature dendritic cells move towards the site of infection. The immature dendritic cells become mature cells when they contact antigen. Therefore,
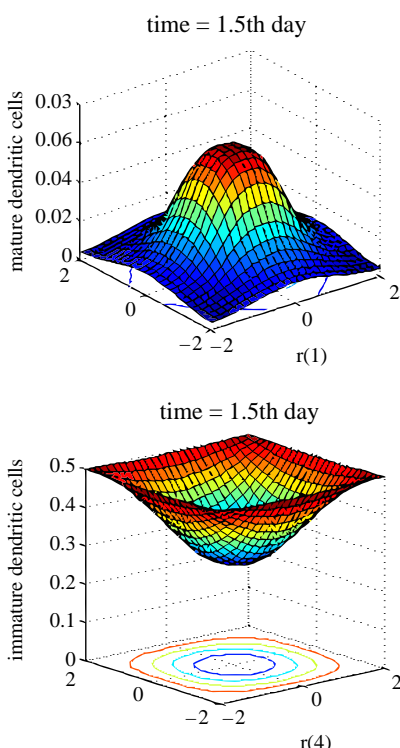
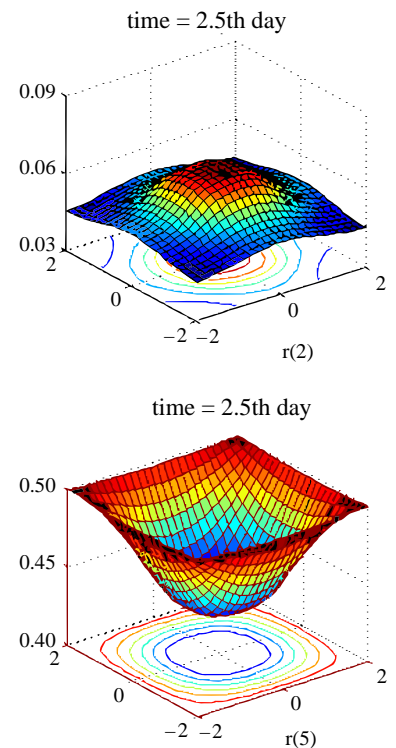

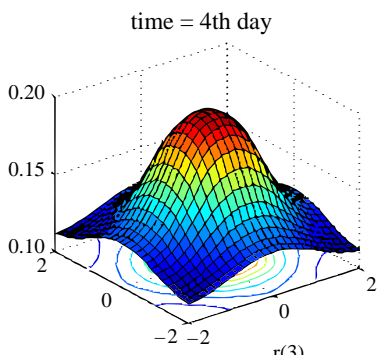

r(3)

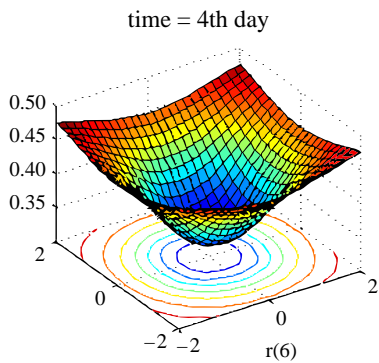

Figure 10. Plots $r(1)$ to $\mathrm{r}(3)$ characterize the evolution of the density of mature dendritic cells, and $r(4)$ to $r(6)$ characterize the one for immature dendritic cells in response to a chronic infection. 

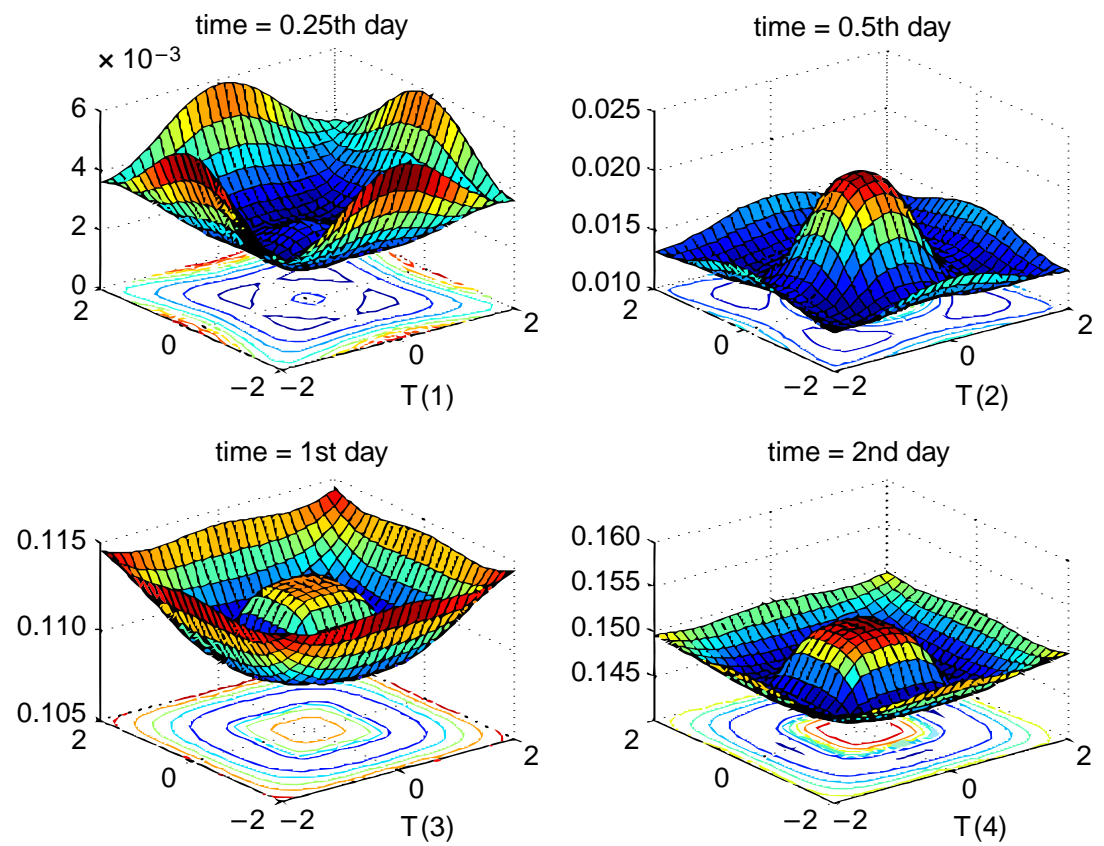

Figure 11. Plots T(1) to T(4) show the evolution of the density of effector and regulatory $\mathrm{T}$ cells.

the distributions of the density of immature and mature dendritic cells have complementary geometries.

Figure 11 focuses on the evolution of $\mathrm{T}$ cell density during chronic infection. We observe that the density of $\mathrm{T}$ cells at the site of infection continuously increases over time. The density of $\mathrm{T}$ cells also increases at the boundary (lymph nodes), however the infection site quickly becomes the most concentrated site of T cell activity.

\subsection{Formation of granuloma}

Granuloma formation is an outcome of a well-regulated immune response. In this section, we explore the distribution of cell types within the infection site during both chronic and acute infections. We also introduce nonsymmetric source functions for antigen/PAMP and observe that granulomas form at the appropriate, now non-central location.

A granuloma has the basic structure of macrophages surrounded by lymphocytes [27], T cells in our model. Figures 12 and 13 focus on distribution of immune cells responding to acute and chronic infection, respectively. In Figure 12, a nascent granuloma-like structure appears during the first four days, but it quickly dissipates once antigen levels decay to zero. The disruption of structure by day 10 is not merely a consequence of departing immune cells, since the density of macrophages and $\mathrm{T}$ cells is still high at this time (see Figure 7).

In Figure 13, the granuloma structure is stable. In fact, the size of the granuloma is stable from about the first day, although $\mathrm{T}$ cells will be increasing in number for several days to come. Corresponding to the temporal dynamics shown in Figure 4, a stable structure of macrophages and T cells is achieved by about day 10 . 

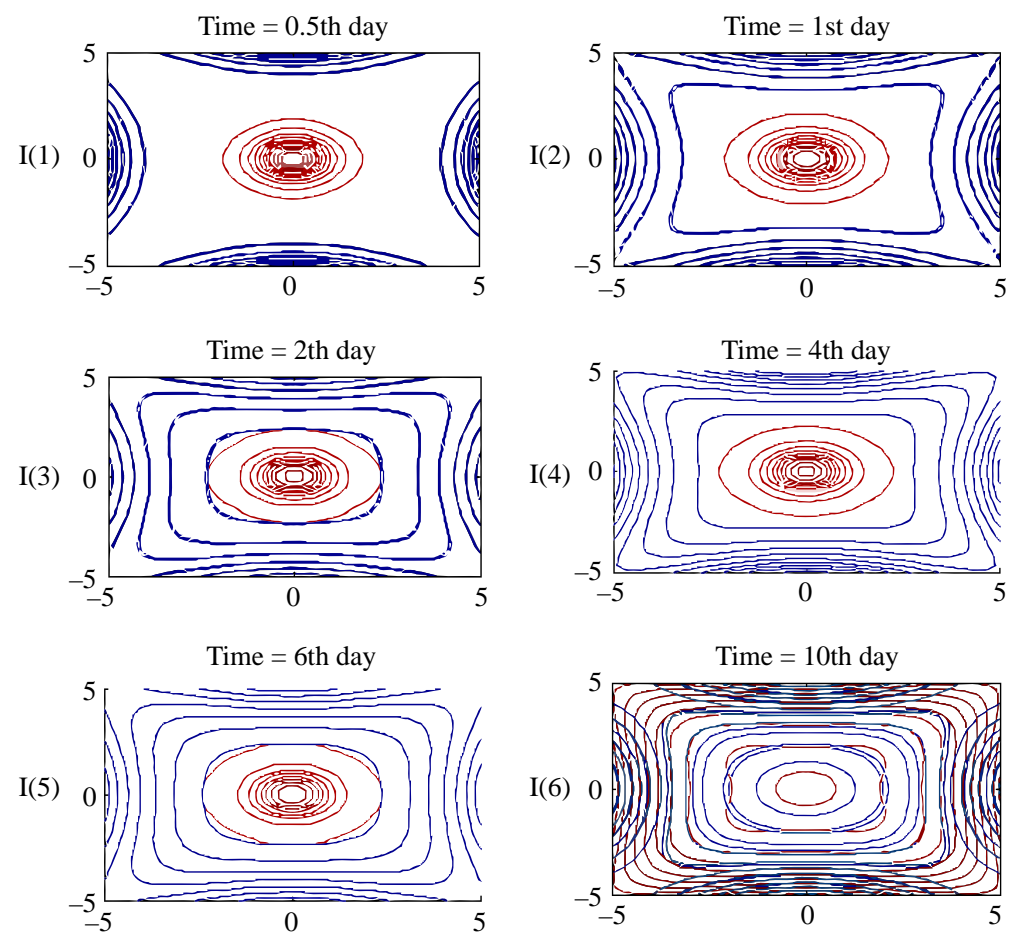

Figure 12. Plots 1(1) to 1(6) show the process of formation of the granuloma for an acute infection. The red curves represent the level sets of macrophage while the blue curves represent the level sets of $\mathrm{T}$ cells.

Finally, we prescribe a non-symmetric source for antigen/PAMP

$$
g(x, t)= \begin{cases}k_{i} & x \in \bigcup_{i=1}^{3} \Omega_{i} \\ 0 & \text { otherwise }\end{cases}
$$

where $k_{i}=2^{i}$ for $i=1,2,3$; and $\Omega_{i} \subset \Omega$ are the small disks in the tissue $\Omega$ such that

$$
\begin{aligned}
& \Omega_{1}=B\left(x_{0}^{1}, r_{2}\right)=B((1,-1), 0.25), \\
& \Omega_{2}=B\left(x_{0}^{2}, r_{2}\right)=B((-2,2), 0.25), \\
& \Omega_{3}=B\left(x_{0}^{3}, r_{3}\right)=B((0,0), 0.25) .
\end{aligned}
$$

Notice that granuloma is gradually formed in the neighborhoods of infection sites $\Omega_{3}$ and $\Omega_{2}$ (refer to $\mathrm{p}(3)$ and $\mathrm{p}(4)$ in Figure 14).

\section{Discussion and conclusions}

Equipped with sophisticated tools such as a highly efficient, convergent numerical scheme and a homogenization method, we have developed a spatial-temporal mathematical model to capture some fundamental aspects of the immune response to antigen (foreign 

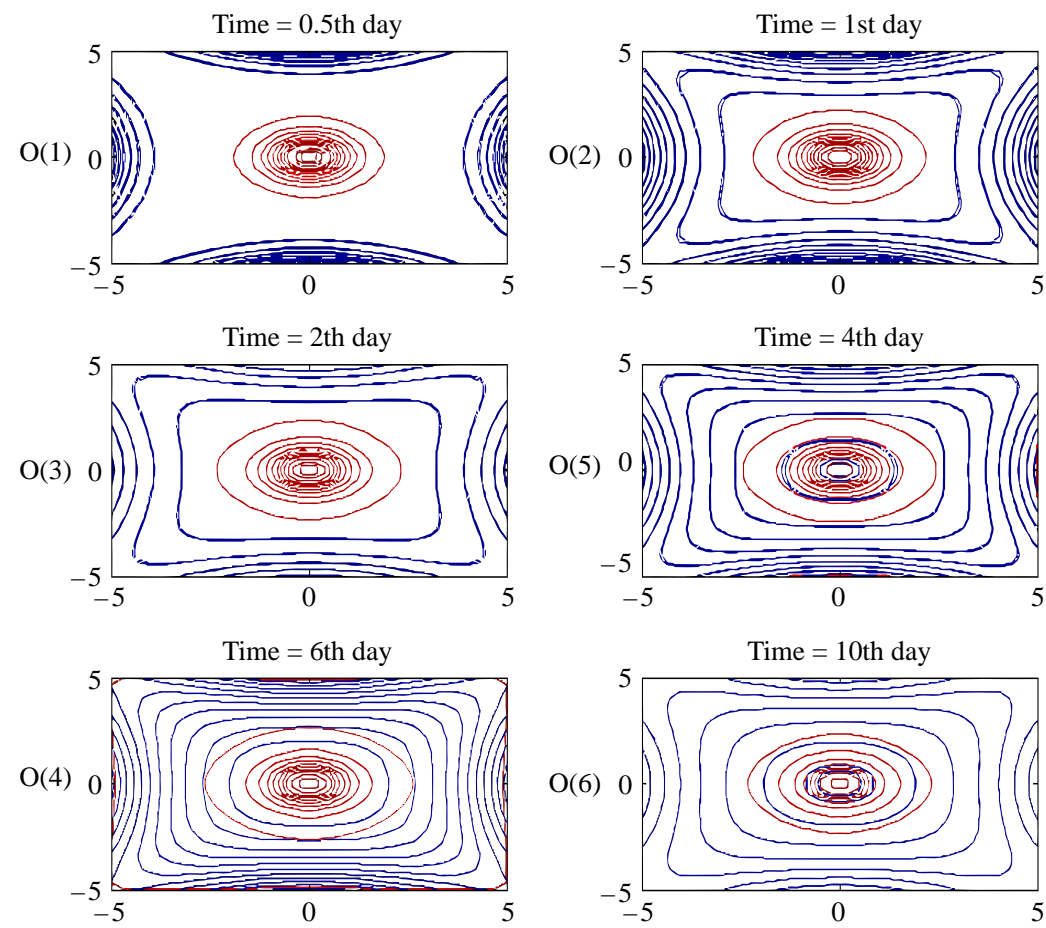

Figure 13. Plots o(1) to o(6) show the process of formation of the granuloma for chronic infection.
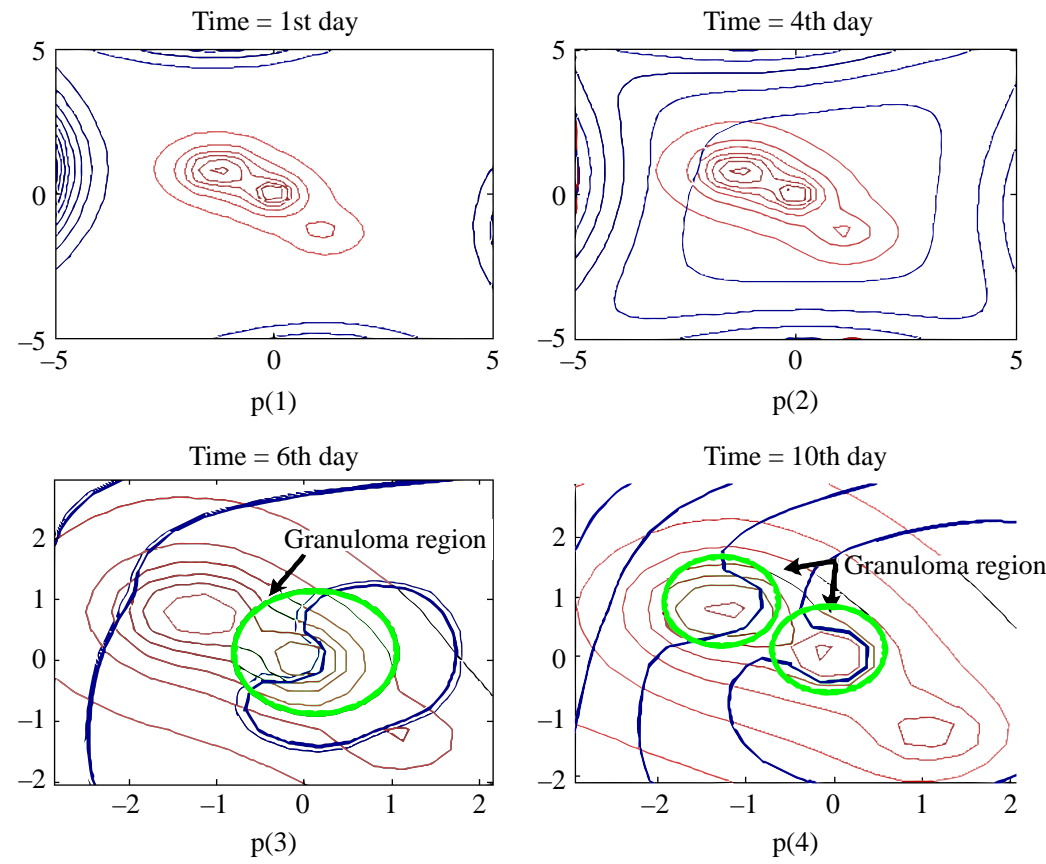

Figure 14. Plots $\mathrm{p}(1)$ to $\mathrm{p}(4)$ show the process of formation of the granuloma for a chronic infection with non-symmetric initial value of antigens in the infectious tissue. Green circles highlight granuloma regions. 
pathogen). System (1)-(10) is a minimal model including only fundamental immune agents. The necessity of dendritic cells and regulatory $\mathrm{T}$ cells for appropriate immune regulation was verified in numerical experiments in Section 5 and is consistent with our current understanding of their central roles in immunology. We have found that the model can account for (1) antigen recognition, (2) an innate immune response (neutrophils and macrophages), (3) an adaptive immune response (T cells), 4) the elimination of antigen and subsequent resolution of the immune response (acute infection) and (5) equilibrium of the immune response to the presence of persistent antigen (chronic infection) and the formation of an immunologically relevant granuloma.

Prior mathematical models of the immune system, such as classical works by [50,55], focus mainly on temporal population dynamics. Besides density/population dynamics, we consider the spatial motilities of the immune agents as a crucial factor. By careful consideration of diffusion and chemotaxis (i.e. convection) rates based upon homogenization, we are able to examine particular dynamics such as regulatory and delay effects. Incorporating the spatial factor makes the arguments for some aspects of the immune response considerably clearer. For example, the delay effect on peak time of T cells is not simply a 'time delay function' but is affected by both spatial diffusion and the direction switch of maturing dendritic cells. The mathematical formulation of a switch of dendritic cells from immature to mature phase upon contact with antigen and an accompanying change in the direction of cell movement is a sophisticated type of signal transduction.

In the model, regulatory mechanisms (Figure 3) are modelled by threshold functions. Neutrophil recruitment and dendritic cell maturation are modelled as simple unidimensional threshold functions. As discussed in Section 2.2, the presence of apoptotic neutrophils and activated macrophages signal the beginning of a successful immune response, after which there is an increasing reliance on macrophage rather than neutrophil scavengers [49]. We use a simple threshold function that blocks neutrophil recruitment when macrophage-produced cytokine $C M$ exceeds a constant threshold $\epsilon_{\mathrm{N}}$. A similar threshold function is used to limit maturation of dendritic cells, so that maturation only occurs when antigen levels exceed $\epsilon_{\mathrm{DE}}$, reflecting the fact that dendritic cells do not activate unless confronted with enough antigen [79]. Regulatory $\mathrm{T}$ cell recruitment and macrophage activation are the most highly regulated processes in our model. As per Equation (6), we see that regulatory $\mathrm{T}$ cells migrate into the infection site only when cytokine produced by effector $\mathrm{T}$ cells, which also functions to promote regulatory $\mathrm{T}$ cell survival, has achieved sufficient mass at the boundary. To sense the state of the infection, regulatory $\mathrm{T}$ cell recruitment also demands that total antigen load at the boundary is not too high. Resting macrophages process the signals from effector and regulatory $\mathrm{T}$ cells as well as pathogen in order to determine their activation status. Equation (8) summarizes the on/off switch utilized by macrophages, showing that effector T cell cytokine $C T$ must exceed an antigen-dependent threshold and regulatory $\mathrm{T}$ cell cytokine $C T_{\text {reg }}$ must not exceed an antigen-dependent threshold, both simultaneously, in order for resting macrophages to activate. In the first case local antigen/PAMP concentration raises the critical threshold, but lowers it in the second.

Our current results do not model a specific type of regulatory $\mathrm{T}$ cell or suggest what regulatory $\mathrm{T}$ cell is most important or responsible for specific disease. We do verify the hypothesis that regulatory $\mathrm{T}$ cells are important to control proinflammatory events and maintain an appropriate relationship between antigen load and the macrophage response, even in our most minimal immune model. 
Acute and chronic antigen exposures are the fundamental types of antigen delivery at infection sites. Our model matches the immunological realities of these disparate conditions reasonably well. We may expect different outcomes using different scales of antigen exposure and it will be interesting to determine if our model will reflect known immunological outcomes under additional scenarios.

Our current model is purposefully simplified and does not include some important immune factors, such as, memory T cells, antibodies (B cells), NK cells, CD8 T cells, etc. The underlying principle we have used to simplify the immune system, is to avoid specific agents and consider instead the fundamental functions of the immune response: (1) antigen recognition, (2) communication, (3) movement and (4) effector function (activation vs. inhibition). A PDE model that captures these generalities is stable. Any additional factors or specifics of the immune response, which increase the granularity of the model, should fall into one of these four categories that are described mathematically in our current model.

In this paper, we determined a mathematical relationship between activation and deactivation of macrophages (the effector response) and regulation of the system (via T cells) through a signal of infection status (antigen load). This general interpretation of immune system function is consistent with other general models that have proposed that antigen load determines the $\mathrm{T}$ cell response through a Tunable Activation Threshold [22] and that antigen levels in the secondary lymph node organs determine the phenotype of the immune response [79]. In our PDE model, we have considered cell-cell communication and cell movement as the fundamentals to an appropriate spatial distribution of the immune response. Numerical simulation for this model is sophisticated and stable. Future work will refine the model and determine the ability of the model to predict relevant biological responses under varying antigen loads that mimic disease states. We will then have the opportunity to study different hypotheses about regulatory networks in silico and then test these hypotheses in vivo.

\section{Acknowledgements}

The authors are grateful to Y. Liu and P. Sacks for their numerous assists on parameter estimates and numerical simulations. B. Su is partially supported by NSF Grant Nos. DMS 0406014 and DMS 0708479 .

\section{References}

[1] A. Ariel and N.C. Serhan, Resolvins and protectins in the termination program of acute inflammation, Trends Biochem. Sci. 28(4) (2007), pp. 176-183.

[2] A. Aderem and D.M. Underhill, Mechanisms of phagocytosis in macrophages, Annu. Rev. Immun. 17 (1999), pp. 593-623.

[3] A. Andoh et al., Cytokine regulation of chemokine (IL-8, MCP-1, and RANTES) gene expression in human pancreatic periacinar myofibroblasts, Gastroenterology 119(1) (2000), pp. 211-219.

[4] S. Akira, TLR signaling, Curr. Top. Microbiol. 311 (2006), pp. 1-16.

[5] C. Asseman et al., An essential role for interleukin 10 in the function of regulatory $T$ cells that inhibit intestinal inflammation, J. Exp. Med. 190(7) (1999), pp. 995-1004.

[6] A.K. Bahl and J.C. Foreman, Stimulation and release of interleukin-1 from peritoneal macrophages of the mouse, Inflam. Res. 42(154) (1994), p. 158.

[7] M.H. Beers, R.S. Porter, and T.V. Jones, The Merck Manual, 18th edition, Merck \& Co., Inc., 2006.

[8] Y. Belkaid et al., $C D 4^{+} C D 25^{+}$regulatory $T$ cells control Leishmania major persistence and immunity, Appl. Opt. 420 (2002), pp. 502-507.

[9] S.B. Brown and J. Savill, Phagocytosis triggers macrophage release of fas ligand and induces apoptosis of bystander leukocytes, J. Immunol 162 (1999), pp. 480-485. 
[10] L. da Fontoura Costa, L.C. Cintra, and D. Schubert, An integrated approach to the characterization of cell movement, Cytometry Part A, 68A (2005), pp. 92-100.

[11] D.M. Catron et al., Visualizing the first $50 \mathrm{~h}$ of the primary immune response to a soluble antigen, Immunity 21(3) (2004), pp. 341-347.

[12] D. Cioranescu and J. Saint Jean Paulin, Homogenization of Reticulated Structures, Springer-Verlag, New York, 1999.

[13] C. D'Amico et al., Uncoupling of inflammatory chemokine receptors by IL-10: generation of functional decoys, Nat. Immunol. 1 (2000), pp. 387-391.

[14] E.J. Ehlert et al., Down-regulation of neutrophil functions by the ELR1 CXC chemokine platelet basic protein, Blood 96(9) (2000), pp. 2965-2972.

[15] V.A. Fadok et al., Macrophages that have ingested apoptotic cells in vitro inhibit proinflammatory cytokine production through autocrine/paracrine mechanisms involving TGF- $\beta, P G E 2$, and PAF, J. Clin. Invest. 101(4) (1998), pp. 890-898.

[16] S. Felder and Z Kam, Human neutrophil motility: Time-dependent three-dimensional shape and granule diffusion, Cell Motil. Cytoskeleton 28(4) (1994), pp. 285-302.

[17] F. Filbet, A finite volume scheme for the Patlak-Keller-Segel chemotaxis model, Numerische Mathematik 104 (2006), pp. 457-488.

[18] D. Gammack, C.R. Doering, and D.E. Kirschner, Macrophage response to Mycobacterium tuberculosis infection, J. Math. Biol. 48 (2004), pp. 218-242.

[19] R.N. Germain and M.K Jenkins, In vivo antigen presentation, Curr. Opin. Immunol. 16 (2004), pp. $120-125$.

[20] A.V. Gett and P.D. Hodgkin, Cell division regulates the T cell cytokine repertoire, revealing a mechanism underlying immune class regulation, Immunology 95(16) (1998), pp. 9488-9493.

[21] B. Gilbertson, J. Zhong, and C. Cheers, Anergy, IFN- $\alpha$ production, and apoptosis in terminal infection of mice with Mycobacterium avium, J. Immunol. 163 (1999), pp. 2073-2080.

[22] Z. Grossman et al., Concomitant regulation of $T$ cell activation and homeostasis, Nat. Rev. Immunol. 4 (2004), pp. 387-395.

[23] C. Haslett, Granulocyte apoptosis and its role in the resolution and control of lung inflammation, Am. J. Respir. Crit. Care Med. 160(5) pt 2 (1999), pp. 25-11.

[24] C. Haurie, C.D. Dale, and C.M. Mackey, Cyclical neutropenia and other periodic hematological disorders: a review of mechanisms and mathematical models, Blood 92(8) (1998), pp. 2629-2640.

[25] B.O. Haglund et al., Effect of SDS micelles on rhodamine-B diffusion in hydrogels, J. Chem. Educ. 72 (1996), pp. 889-893.

[26] A.C. Henderson et al., How does airway inflammation modulate asthmatic airway constriction? An antigen challenge study, J. Appl. Physiol. 95(2) (2003), pp. 873-882.

[27] C.A. Janeway et al., (eds), Immunobiology: The Immune System in Health and Disease, Garland Science Publishing, New York, 2005.

[28] Y. Jiang, A.H. Liu, and K.S. Zhao, Studies on the flow and distribution of leukocytes in mesentery microcirculation of rats, W. J. Gastroenterol. 5(3) (1999), pp. 231-234.

[29] H. Jiang and L. Chess, An integrated view of suppressor T cell subsets in immunoregulation, J. Clin. Invest. 114(9) (2004), pp. 1198-1208.

[30] M.T. Kasaian, K.A. Leite-Morris, and C.A. Biron, The role of $\mathrm{CD}^{+}$cells in sustaining lymphocyte proliferation during lymphocytic choriomeningitis virus infection, J. Immunol. 146(6) (1991), pp. $1955-1963$.

[31] A.T. Kamath et al., The development, maturation, and turnover rate of mouse spleen dendritic cell populations, J. Immunol. 165(6762) (2000), p. 6770.

[32] K.F. Keller and L.A. Segel, Initiation of slime mold aggregation viewed as an instability, J. Theor. Biol. 26 (1970), pp. 399-415.

[33] O. Kim, D. Levy, and P. Lee, Modeling regulatory mechanisms in the immune system, J. Theor. Biol. 246 (2007), pp. 33-69.

[34] B. Knoechel et al., Sequential development of interleukin 2-dependent effector and regulatory $T$ cells in response to endogenous systemic antigen, J. Exp. Med. 202 (2005), pp. 1375-1386.

[35] D.A. Lauffenburger and J.J. Linderman, Receptors: Models for Binding, Trafficking, and Signaling, Oxford University Press, New York, 1993.

[36] A. Lanzavecchia, Mechanisms of antigen uptake for presentation, Curr. Opin. Immunol. 8(3) (1996), pp. $348-354$.

[37] A. Lanzavecchia and F. Sallusto, Regulation of T cell immunity by dendritic cells, Cell 106 (2001), pp. $263-266$.

[38] Y.J. Liu et al., Parameter identification and numerical simulation of chemotaxis equations in PatlakKeller-Segel Model, preprint.

[39] Y.J. Liu, B. Su, and W. Zhou, Numerical approximation of the chemotaxis model by central scheme on overlapping cells with adaptive meshes, in preparation.

[40] A. Marrocco, Numerical simulation of chemotactic bacteria aggregation via mixed finite elements, ESAIM: Math. Mod. Num. Analysis 37 (2003), pp. 617-630.

[41] P. Matzinger, The danger model: A renewed sense of self, Science 296 (2000), pp. 301-305. 
[42] L.C. Meagher et al., Opposing effects of glucocorticoids on the rate of apoptosis in neutrophilic and eosinophilic granulocytes, J. Immunol. 156(11) (1996), pp. 4422-4428.

[43] R. Medzhitov and C. Janeway, Innate immune recognition: Mechanisms and pathways, Immunol. Rev. 173 (2000), pp. 89-97.

[44] T.R. Mempel, S.E. Henrickson, and U.H. von Andrian, T-cell priming by dendritic cells in lymph nodes occurs in three distinct phases, Nature 427 (2004), pp. 154-159.

[45] T.R. Mempel et al., In vivo imaging of leukocyte trafficking in blood vessels and tissues, Curr. Opin. Immunol. 16 (2004), pp. 406-417.

[46] P.V. Moghe, R.D. Nelson, and R.T. Tranquillo, Cytokine-stimulated chemotaxis of human neutrophils in a 3-D conjoined fibrin gel assay, J. Immunol. Methods 180 (1995), pp. 193-211.

[47] H. Mohri et al., Increased turnover of T lymphocytes in HIV-1 infection and its reduction by antiretroviral therapy, J. Exp. Med. 194(9) (2001), pp. 1277-1287.

[48] M.J. Miller et al., Autonomous T cell trafficking examined in vivo with intravital two-photon microscopy, J. Immunol. 100(5) (2003), pp. 2604-2609.

[49] C. Nathan, Neutrophils and immunity: Challenges and opportunities, Nat. Rev. Immunol. 6 (2006), pp. $173-182$

[50] M.A. Nowak and R.M. May, Mathematical biology of HIV infection: antigenic variation. and diversity threshold, Math. Biosci. 106 (1991), pp. 1-21.

[51] T. Okada et al., Antigen-engaged B cells undergo chemotaxis toward the T zone and form motile conjugates with helper T cells, PLoS Biol. 3(6) (2005), pp. 1047-1061.

[52] M.R. Owen and J.A. Sherratt, Pattern formation and spatiotemporal irregularity in a model for macrophage-tumor interactions, J. Theor. Biol. 189 (1997), pp. 63-80.

[53] M.R. Owen and J.A. Sherratt, Mathematical modeling of macrophage dynamics in tumours, Math. Models Methods Appl. 9 (1999), pp. 513-539.

[54] G. Pap et al., Self-regulation of neutrophils during phagocytosis is modified after severe tissue injury, Int. J. Mol. Med. 17(24) (2006), pp. 649-654.

[55] A.S. Perelson, Modeling the Interaction of the Immune System with HIV, Mathematical and Statistical Approaches to AIDS Epidemiology (Lecture Notes in Biomathematics), in C. Castillo-Chavez, ed., SpringerVerlag, New York, 1989, pp. 350-370.

[56] L.E. Racoosin and A.J. Swanson, M-CSF-induced macropinocytosis increases solute endocytosis but not receptor-mediated endocytosis in mouse macrophages, J. Cell Sci. 102 (1992), pp. 867-880.

[57] A. Rot and U.H. von Andrian, Chemokines in innate and adaptive host defense: basic chemokinese grammar for immune cells, Annu. Rev. Immunol. 22 (2004), pp. 891-928.

[58] M. Rabinovitch, Professional and non-professional phagocytes: An introduction, Trends Cell Biol. 5 (1995), pp. 85-87.

[59] G.D. Russell, Who puts the tubercle in the tuberculosis?, Nat. Rev. Micro. 5 (2007), pp. 39-47.

[60] S.I. Rubinow and J.L. Lebowitz, A mathematical model of neutrophil production and control in normal man, J. Math. Biol. 1(3) (1975), pp. 187-225

[61] F. Sallusto et al., Dendritic cells use macropinocytosis and the mannose receptor to concentrate macromolecules in the major histocompatibility complex class II compartment: Downregulation by cytokines and bacterial products, J. Exp. Med. 182 (1995), pp. 389-400.

[62] S. Sakaguchi et al., Immunologic self-tolerance maintained by activated T cells expressing IL-2 receptor a-chains (CD25). Breakdown of a single mechanism of self-tolerance causes various autoimmune diseases, J. Immunol. 155(3) (1995), pp. 1151-1164.

[63] H. Scott, J. Chan, and J. Flynn, Chemokines and tuberculosis, Cytokine Growth Factor Rev. 14 (2003), pp. 467-477.

[64] N.C. Serhan et al., Resolution of inflammation: State of the art, definitions and terms, FASEB J. 21 (2007), pp. $325-332$

[65] S. Sozzani et al., The signal transduction pathway involved in the migration induced by a monocyte chemotactic cytokine, J. Immunol. 147 (1991), pp. 2215-2221.

[66] J. Sprent, Lifespans of naïve, memory and effector lymphocytes, Curr. Opin. Immunol. 5 (1993), pp. $433-438$.

[67] D. Sredni-Kenigsbuch, T. Kambayashi, and G. Strassmann, Neutrophils augment the release of TNF- $\alpha$ from LPS-stimulated macrophages via hydrogen peroxide, Immunol. Lett. 71(2) (2000), pp. 97-102.

[68] B. Su and E. DiBenedetto, Homogenization in the diffusion of IP3 from cell membrane to endoplasmic reticulum, Preprint.

[69] S. Suvas and T.B. Rouse, $\mathrm{T}_{\text {reg }}$ control of antimicrobial T cell responses, Curr. Opin. Immunol. 18 (2006), pp. 344-348

[70] L. Tartar, Nonhomogeneous Media and Vibration Theory, in E. Sanchez-Palencia, ed., Springer-Verlag, Berlin, 1980, Appendix.

[71] J.W. Thomas, Numerical Partial Differential Equations: Finite Difference Methods, Springer-Verlag, New York, 2004

[72] R.T. Tranquillo and D.A. Lauffenburger, Definition and measurement of cell migration coefficients in Biological Motion (Lecture Notes in Biomathematics 89), in W. Alt and G. Hoffmann, eds., Springer-Verlag, New York, 1990, pp. 475-486. 
[73] Y. Vanloubbeeck, J. Hostetter, and D.E. Jones, The biology of dendritic cells and their potential use in veterinary medicine, Anim. Health Res. Rev. 4 (2003), pp. 131-142.

[74] U.H. von Andrian and T.R. Mempel, Homing and cellular traffic in lymph nodes, Nat. Rev. Immunol. 3 (2003), pp. 867-878.

[75] H. von Boehmer, Mechanisms of suppression by suppressor T cells, Nature. Immunol. 6 (2005), pp. $338-344$.

[76] J.E. Wigginton and D.E. Kirschner, A model to predict cell-mediated immune regulatory mechanisms during human infection with mycobacterium tuberculosis, J. Immunol. 166 (2001), pp. 1951-1967.

[77] A.M. Williams et al., Chemokine regulation of neutrophil function in surgical inflammation, Archiv. Surg. 134(12) (1999), pp. 1360-1366.

[78] Z. Xing, Current understanding of macrophage type 1 cytokine responses during intracellular infections, Histol. Histopathol. 15(1) (2000), pp. 199-205.

[79] R.M. Zinkernagel, Localization dose and time of antigens determine immune reactivity, Semin. Immunol. 12 (2000), pp. $163-171$ 


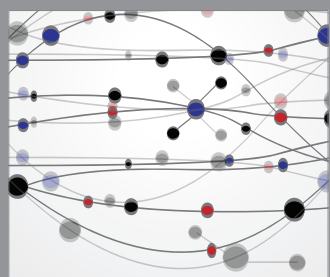

The Scientific World Journal
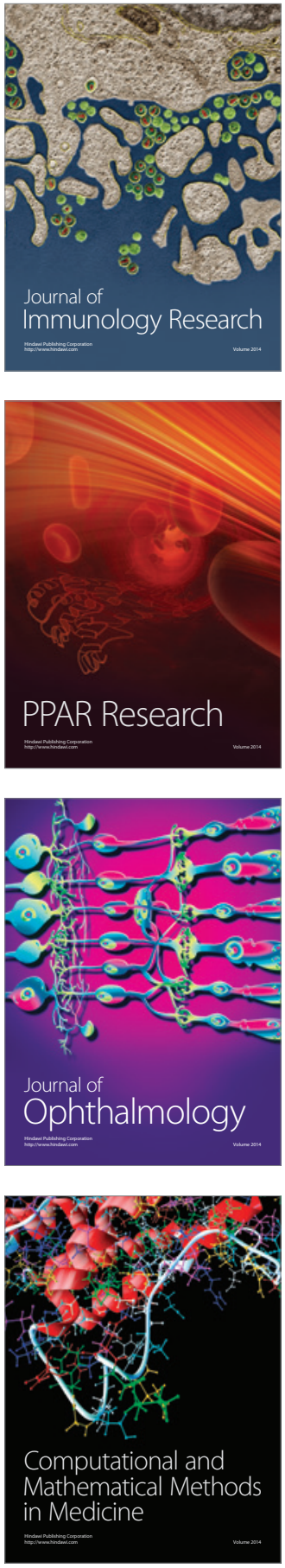

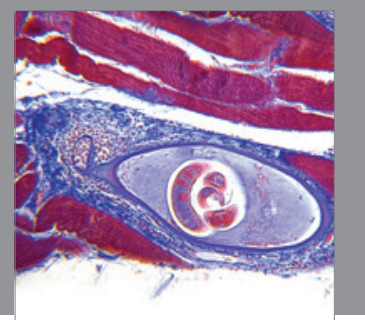

Gastroenterology

Research and Practice
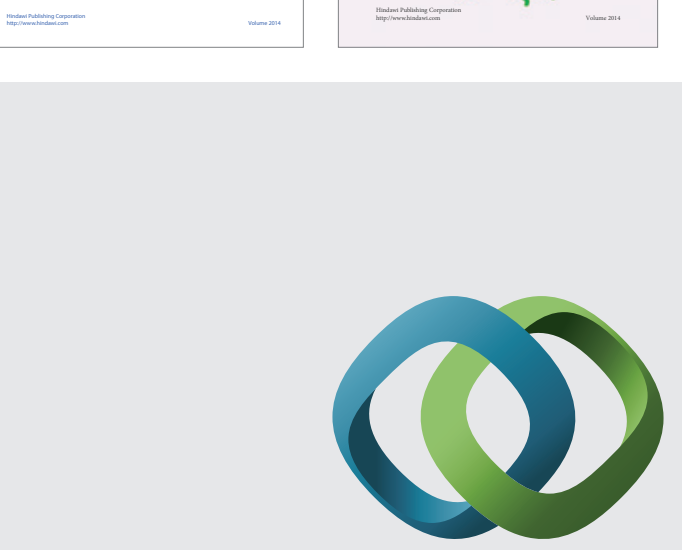

\section{Hindawi}

Submit your manuscripts at

http://www.hindawi.com
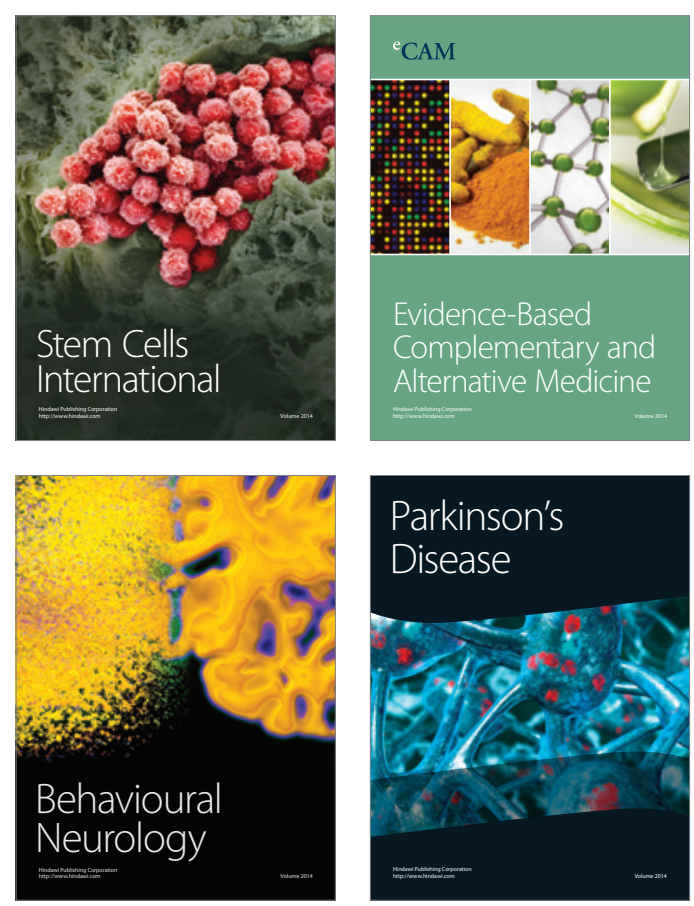

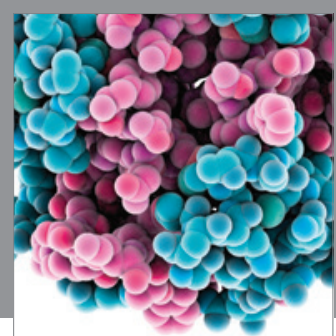

Journal of
Diabetes Research

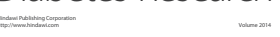

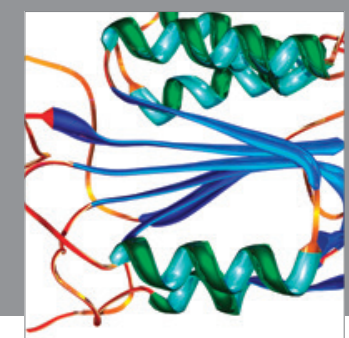

Disease Markers
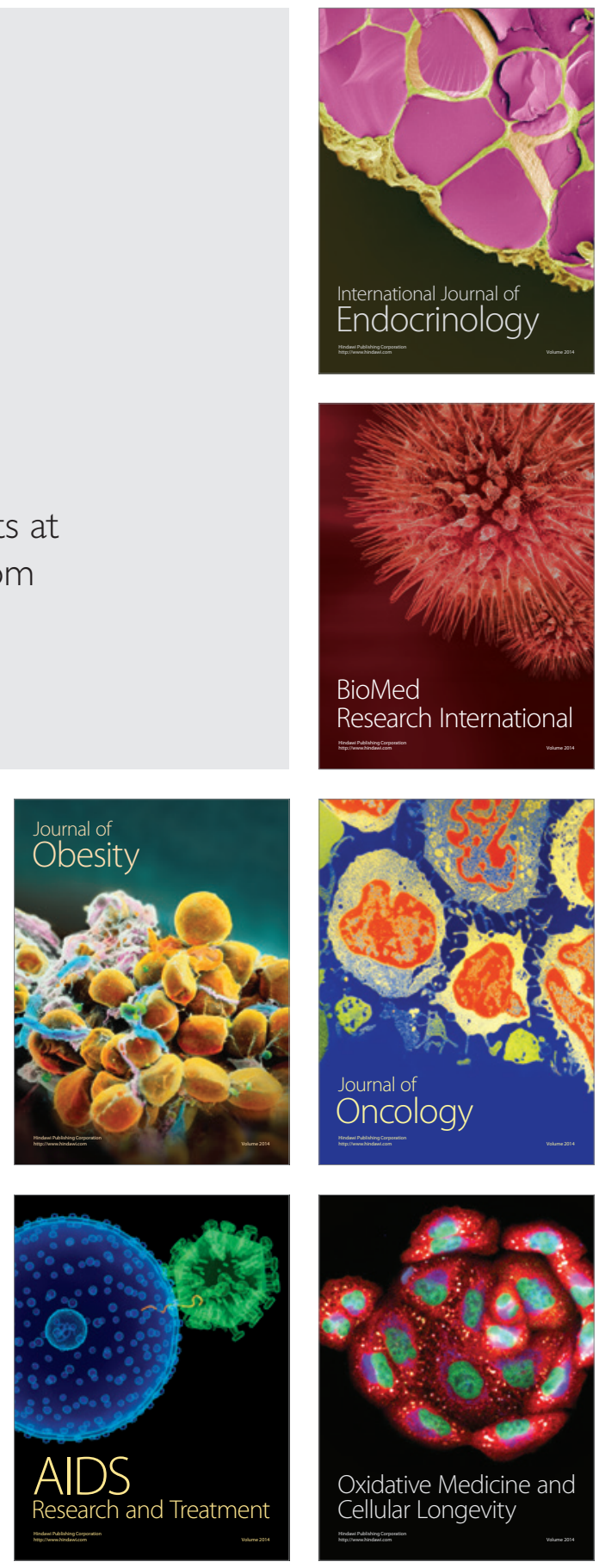